\title{
Using radio astronomical receivers for molecular spectroscopic characterization in astrochemical laboratory simulations: A proof of concept ${ }^{\star}$
}

\author{
I. Tanarro' ${ }^{1}$, B. Alemán ${ }^{2}$, P. de Vicente ${ }^{3}$, J. D. Gallego ${ }^{3}$, J. R. Pardo ${ }^{2}$, G. Santoro ${ }^{4}$, K. Lauwaet ${ }^{4}$, F. Tercero ${ }^{3}$, \\ A. Díaz-Pulido ${ }^{3}$, E. Moreno ${ }^{2}$, M. Agúndez ${ }^{2}$, J. R. Goicoechea ${ }^{2}$, J. M. Sobrado ${ }^{5}$, J. A. López ${ }^{3}$, L. Martínez ${ }^{4}$, \\ J. L. Doménech ${ }^{1}$, V. J. Herrero ${ }^{1}$, J. M. Hernández ${ }^{3}$, R. J. Peláez ${ }^{1}$, J. A. López-Pérez ${ }^{3}$, J. Gómez-González ${ }^{3}$, \\ J. L. Alonso ${ }^{6}$, E. Jiménez ${ }^{7}$, D. Teyssier ${ }^{8}$, K. Makasheva ${ }^{9}$, M. Castellanos ${ }^{2}$, C. Joblin ${ }^{10,11}$, \\ J. A. Martín-Gago ${ }^{4}$, and J. Cernicharo ${ }^{2}$
}

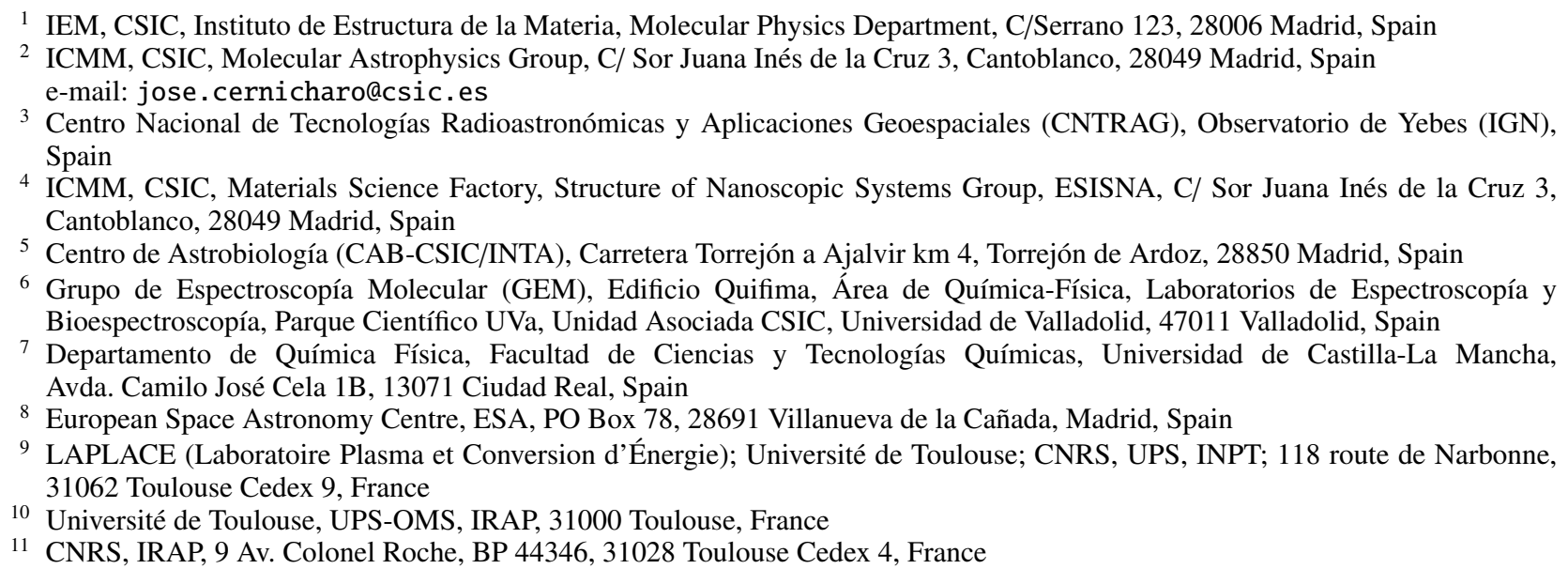

Received 10 April 2017 / Accepted 21 September 2017

\begin{abstract}
We present a proof of concept on the coupling of radio astronomical receivers and spectrometers with chemical reactors and the performances of the resulting setup for spectroscopy and chemical simulations in laboratory astrophysics. Several experiments including cold plasma generation and UV photochemistry were performed in a $40 \mathrm{~cm}$ long gas cell placed in the beam path of the Aries $40 \mathrm{~m}$ radio telescope receivers operating in the $41-49 \mathrm{GHz}$ frequency range interfaced with fast Fourier transform spectrometers providing $2 \mathrm{GHz}$ bandwidth and $38 \mathrm{kHz}$ resolution. The impedance matching of the cell windows has been studied using different materials. The choice of the material and its thickness was critical to obtain a sensitivity identical to that of standard radio astronomical observations. Spectroscopic signals arising from very low partial pressures of $\mathrm{CH}_{3} \mathrm{OH}, \mathrm{CH}_{3} \mathrm{CH}_{2} \mathrm{OH}, \mathrm{HCOOH}, \mathrm{OCS}, \mathrm{CS}, \mathrm{SO}_{2}\left(<10^{-3}\right.$ mbar) were detected in a few seconds. Fast data acquisition was achieved allowing for kinetic measurements in fragmentation experiments using electron impact or UV irradiation. Time evolution of chemical reactions involving $\mathrm{OCS}, \mathrm{O}_{2}$ and $\mathrm{CS}_{2}$ was also observed demonstrating that reactive species, such as CS, can be maintained with high abundance in the gas phase during these experiments.
\end{abstract}

Key words. methods: laboratory: molecular - instrumentation: spectrographs - astrochemistry - molecular data

\section{Introduction}

Molecular spectroscopy is the basic tool used to study the chemical, physical, and dynamical evolution of molecular clouds, where stars and their planets form. Radio astronomers use large single dishes or interferometers equipped with very sensitive receivers to detect the thermal emission of the molecules present in these objects. Although volume densities in molecular clouds, mostly corresponding to $\mathrm{H}_{2}$, are rather low, $\sim 10^{2}-10^{5} \mathrm{~cm}^{-3}$, the large size of these objects (typically several parsecs $\sim 10^{19} \mathrm{~cm}$ ), allows column densities to be rather high,

\footnotetext{
^ Movies are available at http://www . aanda.org
}

$\sim 10^{21}-10^{24} \mathrm{~cm}^{-2}$. For a molecular cloud with a column density of $10^{22} \mathrm{H}_{2}$ molecules $\mathrm{cm}^{-2}$ (for example a prestellar core such as TMC1), the column densities of detected polar species vary from $\sim 10^{11}$, for the less abundant ones, to $\sim 10^{18} \mathrm{~cm}^{-2}$ for CO. Using large single-dish telescopes (such as the $30 \mathrm{~m}$ IRAM radio telescope) or interferometers (ALMA, NOEMA), the detection of the weakest and narrowest molecular lines in cold dark clouds, requiring $\sim 20-30 \mathrm{kHz}$ spectral resolution, typically requires a few hours of observing time. On the other hand, the broadest and most intense molecular lines in space are detected in a matter of minutes using frequency resolutions of the order of $1 \mathrm{MHz}$ with the same facilities. 
In order to simulate one of these molecular clouds here on Earth we would have to compress and confine the whole column of gas that a telescope observes within its beam into a gas cell which typically would have a length of the order of one meter. Column densities of the less abundant, but detected, molecules in space $\left(N \simeq 10^{11} \mathrm{~cm}^{-2}\right)$ would translate into partial pressures inside such a chamber in the range $\sim 10^{-8}$ mbar (at $T_{\mathrm{K}} \sim 10 \mathrm{~K}$ ) to $\sim 5 \times 10^{-6}$ mbar (at $T_{\mathrm{K}} \sim 300 \mathrm{~K}$ ). The idea behind this work is that an astronomical receiver placed in front of such a gas cell would detect lines of these low-abundance species in minutes to hours.

Radio astronomical receivers nowadays permit broad instantaneous frequency coverage (several $\mathrm{GHz}$ ) that, in turn, can be fully covered with Fast Fourier Transform Spectrometers (FFTS) providing spectral resolutions as low as a few $\mathrm{kHz}$. Standard techniques for millimeter laboratory spectroscopy use different approaches to provide high sensitivity, broad band coverage, and spectral resolution such as the Chirped pulse Fourier Transform microwave (CHIRP) spectroscopy (Brown et al. 2008; Dian et al. 2008), extended to the millimeter region as presented in Park et al. (2011). However, sensitivity decreases considerably with increasing frequency. Chirped pulse spectroscopy has been applied by various groups to study kinetics and gas compositions (Abeysekera et al. 2014; Gerecht et al. 2011). Although the CHIRP pulse is not a passive method it has been shown that the initial population difference does not change in the linear fast passage regime for weak coupling (weak pulse limit) normally assumed. However, a large change in the population difference within the rapid adiabatic passage regime is observed for the strong coupling regime (strong pulse or high dipole moment). Here, stepwise multi-resonance effects can alter the relative intensities of the observed rotational transitions. In fact, the intensities differ significantly between measurements with chirp-up and chirp-down (Schmitz et al. 2012), which could cause serious difficulties in the estimation of the abundance of the gases in the chamber. Partial pressures, however, can be easily obtained from the measurement of the molecular thermal emission using a radio astronomical receiver, just as radio astronomers do when observing interstellar clouds.

Within the European Research Council Synergy project NANOCOSMOS we proposed using radio astronomical receivers as detectors for molecular spectroscopy and chemical reactivity experiments in a gas cell. The aim of this work is therefore to present our first proof-of-concept and to shed light on the main issues related to the technique. Different experiments have been performed based on similar ideas (Hüberts et al. 2006; Ren et al. 2010; Neumaier et al. 2014; Wehres et al. 2017), in particular for the SWAS satellite for which an OCS gas cell was used (Tolls et al. 2004), the Herschel/HIFI receivers (Higgins et al. 2010; Higgins 2011; Teyssier et al. 2004), and the Swedish Odin satellite that used a gas cell filled with $\mathrm{H}_{2} \mathrm{O}$ (Frisk et al. 2003). Except for the experiment of Wehres et al. (2017), these experimental setups were focussed on the prelaunch characterization of receivers and not on the possibility of using the receivers for laboratory spectroscopy and chemical reactors.

The main advantage of thermal emission with respect absorption techniques (Clark \& De Lucia 1981) is the instantaneous broad band provided by radioastronomical receivers that permits to follow the variation of intensity with time of the emission lines of many molecular species. The main goal of this proof of concept is to show that kinetic simulations (photochemistry, cold plasma, discharges) could be performed addressing chemical compositions similar to those of interstellar clouds.

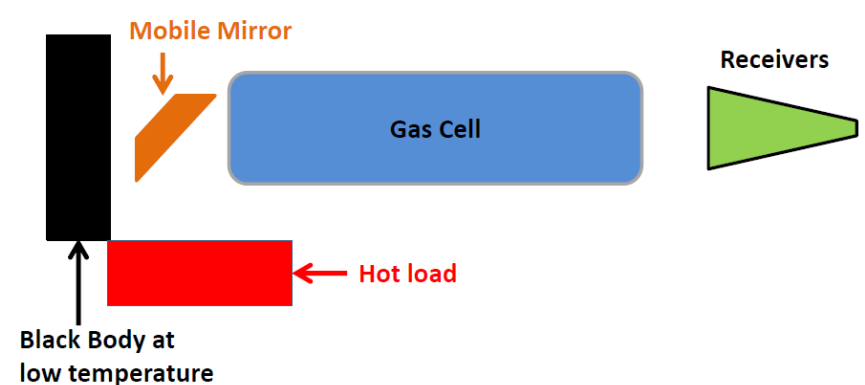

Fig. 1. Basic design concept of the gas cell setup.

This paper is devoted to the development of a gas cell prototype installed in the beam path of the receivers of the $40 \mathrm{~m}$ Aries telescope. It was designed to study the spectroscopy and chemistry of cold plasmas, with or without the action of UV photons. Section 2 describes the experimental setup, the data acquisition system, and its performances. Calibration procedures are explained in Sect. 3 while in Sect. 4 we describe the expected molecular thermal emission in the gas cell. Finally, in Sect. 5 we present the results obtained under static or flow modes with, or without, cold plasma or UV irradiation conditions.

\section{Experimental setup}

\subsection{Basic design}

The basic concept of the gas cell and the detection scheme is depicted in Fig. 1. It will be a chamber of $60 \mathrm{~cm}$ diameter and $100 \mathrm{~cm}$ length that will be equipped with temperature and pressure sensors, a quadrupole mass spectrometer, pipeline entries for the different gases, a cold plasma generator, and UV lamps. An absorber at temperature $T_{\mathrm{c}}$ (microwave absorber embedded in liquid $\mathrm{N}_{2}$ or inside a cryostat at $20 \mathrm{~K}$; hereafter referred to as cold load) will be behind the cell and in the direct optical path of the receivers allowing to measure the thermal molecular emission of the gases inside the chamber (see Sect. 3).

A prototype has been built as described below to demonstrate that radio astronomical receivers can be used for molecular characterization in experimental gas chambers (spectroscopy and/or chemical reactivity). The experiments with the gas cell prototype were carried out by installing it in the beam path of the $40 \mathrm{~m}$ radiotelescope at CNTRAG-IGN (Guadalajara, Spain). Figures 2-4 show the chamber and its installation in the receiver room of the telescope. The prototype chamber is a stainless steel cylinder of $40 \mathrm{~cm}$ length and $25 \mathrm{~cm}$ diameter giving a total volume of 20 liters approximately. It was designed with non-parallel windows to avoid multiple reflections between the two windows during the spectra acquisition with radio astronomical receivers (see Sect. 2.4). The detection system is mounted on a table which holds the $Q$ band high electron mobility transistor (HEMT) receiver, the mirrors and the cold load. The $Q$ band receiver detects molecular emission from rotational transitions in the $41-49 \mathrm{GHz}$ frequency range against the background radiation coming from the cold load (see Figs. 1-4). The gas chamber is located where the gaussian beam narrows to its minimum width to permit the smallest possible size for the windows of the cell. Regarding the number of ports in the chamber, Figs. $2 \mathrm{a}$ and $\mathrm{c}$ show the first and the final versions of the gas cell prototype. The increase in the number of ports allowed us to improve the flow conditions, and to connect a variety of instrumentation. Most of the results presented here were obtained with this final version. 


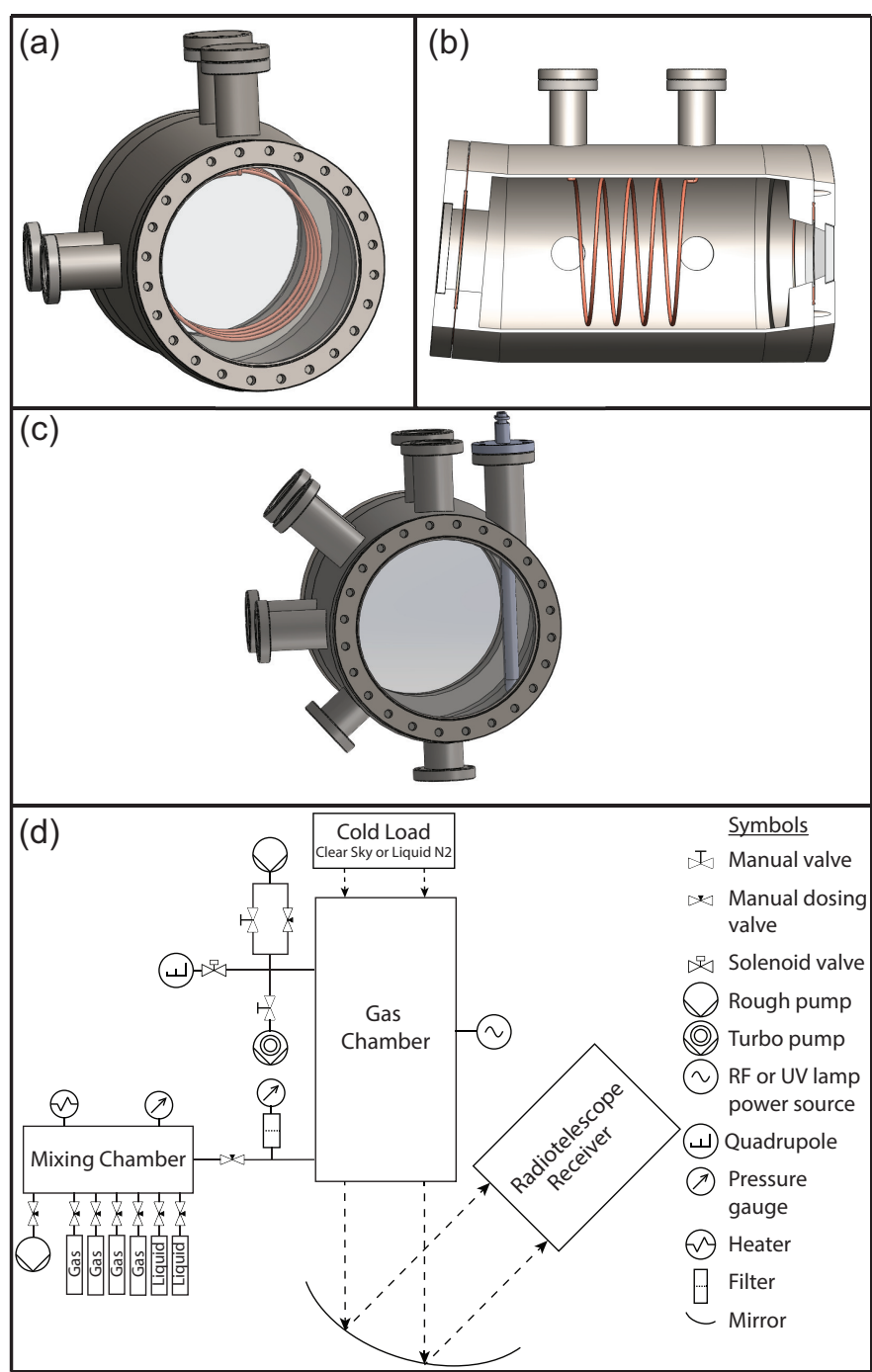

Fig. 2. Design of the gas cell prototype $(a)$, its configuration as plasma reactor $(b)$ and with the UV lamp $(c)$, and experimental set-up showing the technical scheme of the gas mixing, mass spectrometry, and gas phase molecular detection $(d)$.

The gas or gas mixture is injected from a gas mixing chamber equipped with six inlets with manual dosing valves (four for gases and two for liquids) and a $3 \mathrm{~m}^{3} \mathrm{~h}^{-1}$ rotary pump (Pfeiffer Duo $3 \mathrm{M}$ ) that allows pressure control inside the mixing chamber in the range of 0-100 mbar (Fig. 2d). Carbonyl sulfide (OCS) from Air Liquide, $\mathrm{O}_{2}, \mathrm{He}$, and $\mathrm{N}_{2}$ from Praxair all with $>99 \%$ purity in gas phase, and $\mathrm{CS}_{2}, \mathrm{CH}_{3} \mathrm{OH}, \mathrm{CH}_{3} \mathrm{CH}_{2} \mathrm{OH}$ and $\mathrm{HCOOH}$ (Sigma Aldrich $>98 \%$ ) in liquid phase, were used in this work. In order to avoid liquid condensation during the injection, the mixing chamber and injection tube were heated up to $40{ }^{\circ} \mathrm{C}$.

For static regime experiments the gas is injected from the gas mixing chamber through a manual dosing valve until the desired pressure in the range of $0.1-0.5$ mbar is reached. However, to carry out dynamic regime experiments the gas flow and pressure inside the gas chamber were controlled at the outlet by a $30 \mathrm{~m}^{3} \mathrm{~h}^{-1}$ dry scroll pump (Agilent Technologies TriScroll 600). In such conditions, typical gas residence time is approximately $8 \mathrm{~s}$.

The evacuation process between experimental runs is performed with a $250 \mathrm{l} / \mathrm{s}$ turbomolecular pump (Agilent Technologies Twistorr 304FS). Initial pumping stages from atmospheric

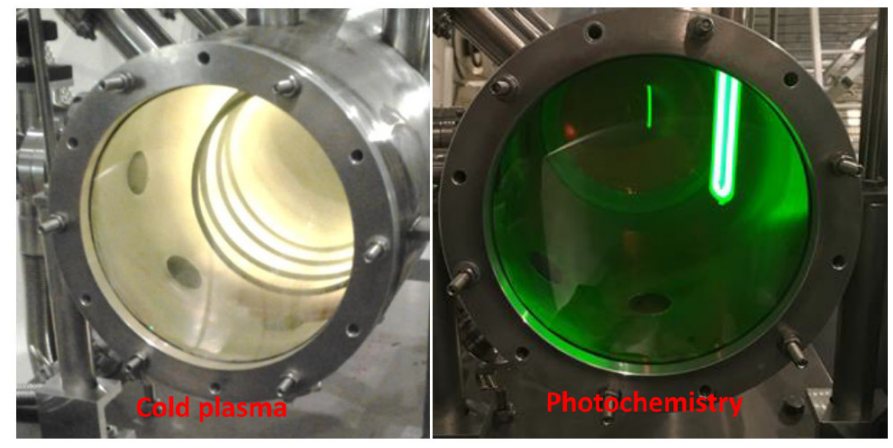

Fig. 3. Reactor with plasma on (left) and the UV lamp (right). The colours of the plasma glow emission and the UV lamp emission are determined by the optical transmission of the brown Upilex windows.

pressure were performed very slowly to prevent the sudden breaking of the thin polymeric windows, which deformed inward markedly and in some cases imploded very loudly. To read the pressure inside the gas chamber in the different vacuum conditions, two different pressure gauges (capacitance manometer Leybold CTR90 for experiments and Agilent Technologies FRG-702 Pirani/cold cathode for the initial vacuum) were used, both coupled to a single independent flange of the gas-cell through a fine mesh filter and a vacuum tee piece (in the final version of the prototype, see Fig. 2c), and both connected to their respective gauge controllers (Leybold Center Two and Agilent Technologies XGS 600, respectively) in order to register the pressure through an in-house developed software routine.

To control the chemical processes during the experiments, a differentially pumped quadrupole mass spectrometer (Pfeiffer Prisma Plus) working in the $0-100$ a.m.u. range is connected to the chamber through a solenoid actuated regulating gas valve (Pfeiffer RME 005 A valve and RVC-300 controller) that limits the flow in order to maintain the adequate working pressure for the quadrupole $\left(5 \times 10^{-6} \mathrm{mbar}\right)$. At this working pressure, a Faraday cup was used as detector to avoid saturation or damage of the also available Secondary Electron Multiplier (SEM) detector. We note that the mass spectrometer does not provide information about unstable species, due either to their recombination in the path to the spectrometer or because their signals coincide with a fragment of a stable species (precursor or product). Furthermore, it cannot discriminate among different species with the same molecular mass.

Depending on the molecular excitation and dissociation process, inductively coupled radio frequency (RF) discharge or UV radiation, different instrumentation was placed inside the chamber. Figures $2 \mathrm{a}$ and $\mathrm{b}$ (cross section view) show the first version of the gas cell with a copper coil (6 mm outside diameter copper tubing, four turns, $10 \mathrm{~cm}$ length and $17 \mathrm{~cm}$ diameter) placed inside the chamber parallel to the primary cylinder axis for low pressure plasma experiments in static or dynamic regime. The $13.56 \mathrm{MHz}$ RF generator (Hüttinger PFG $300 \mathrm{RF}+$ matchbox PFM 1500A) was connected between one of the ends of the $\mathrm{Cu}$ coil (electrically isolated) and the grounded chamber at the minimum feasible distance. The shortest possible wires were used to minimize RF radiation noise. The other end of the coil was grounded. RF powers of 5-100 W were typically applied to sustain the plasma. The coil was refrigerated by a circulating water circuit.

For UV radiation experiments, a vacuum system adapted UV lamp (UVB-100, RBD Instruments) was placed inside the chamber where the interference with the receiver beam path is 

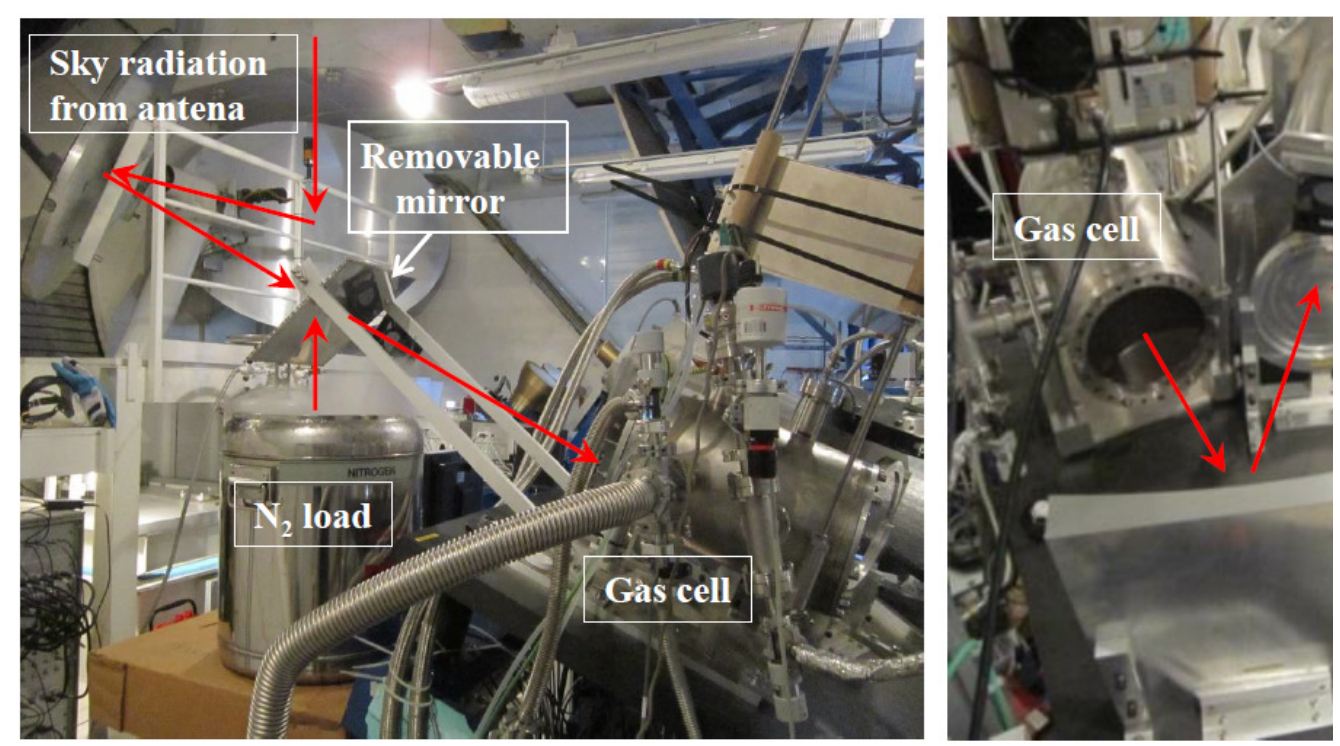

Fig. 4. Prototype of the gas cell installed in the receiver room of the $40 \mathrm{~m} \mathrm{IGN}$ radio telescope. The cell is in the optical axis of the $Q$ band receiver. Most of the observations were performed using the sky emission as cold load (see text). In case of poor weather conditions, the $\mathrm{N}_{2}$ cold load was used.

minimized, as shown in Fig. $2 \mathrm{c}$ for the final version of the chamber. The low-pressure $\mathrm{Hg}$ lamp spectrum exhibits emission lines at $185 \mathrm{~nm}(30 \%)$ and $254 \mathrm{~nm}(70 \%)$ with a total power of $20 \mathrm{~W}$ in the UVC range $(100-280 \mathrm{~nm})$.

\subsection{Data acquisition}

The prototype of the gas cell was installed in the receiver room of the $40 \mathrm{~m}$ IGN radio telescope as shown in Fig. 4. The receiver beam width along the axial axis of the cell (at 1/e intensity level) was always smaller than the diameters of the gas cell windows and the coil, allowing standard radio astronomical observations, if needed, during the experiments (see below and Fig. 5). Three different experimental runs were carried out between October 2015 and November 2016.

Observations were performed with a dual-polarization (left and right circular polarization) single-pixel $45 \mathrm{GHz}$ receiver mounted in the Nasmyth focus. The feed horn, orthomode transducer and HEMT amplifiers of the receiver were cooled at cryogenic temperature $(15 \mathrm{~K})$ in a cryostat. The front end provides an instantaneous bandwidth of $9 \mathrm{GHz}$, from 41 to $50 \mathrm{GHz}$ for each polarization but the intermediate frequency (IF) stage limits it to an instantaneous band of $2 \mathrm{GHz}$. The average noise temperature of the receiver is $45 \mathrm{~K}$ (the input termination temperature should be added to obtain system noise temperature). The design of the IF completely rejects the image frequency and the receiver can be considered of the single side band (SSB) type. Both base bands from the two polarizations, between 0 and $2 \mathrm{GHz}$, were sent using RF-over-fiber optical links to the backends room where the signal was injected into a Fast Fourier Transform Spectrometer with two modules of $2.5 \mathrm{GHz}$ band width and 65536 channels, which provides a frequency resolution of $38.1 \mathrm{kHz}$. Only the first 52429 channels $(2 \mathrm{GHz}$ ) were used in the final spectra. The Allan variance minimum time, based on the previous astronomical observations, is of the order of few minutes.

Calibration was performed using a hot and a cold load after having evacuated the gas cell down to a pressure of approximately $2 \times 10^{-3}$ mbar. It consisted on two phases, one integration of typically $30 \mathrm{~s}$ using a microwave absorber at room

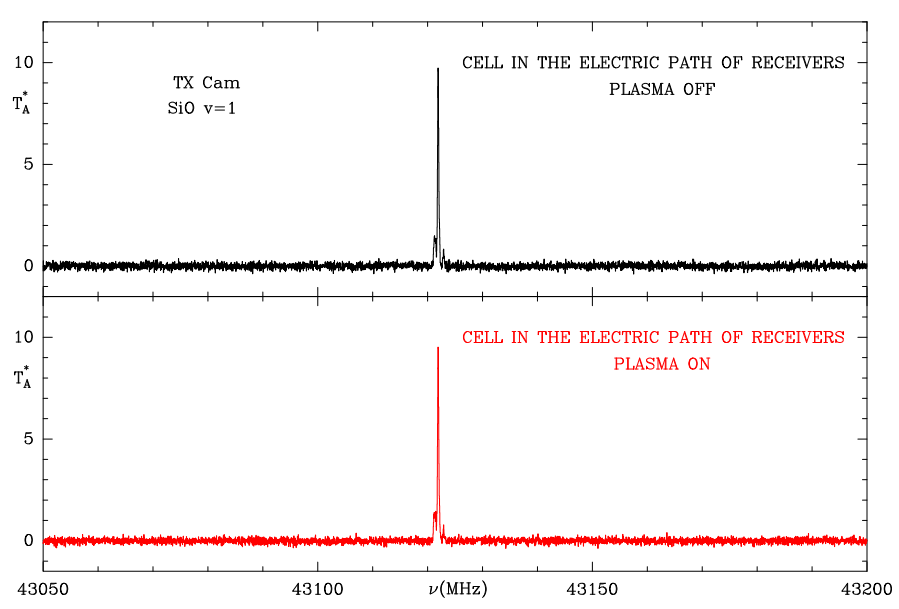

Fig. 5. Observed spectrum with an He plasma on (bottom) and off (top) towards TX Cam. The selected frequency corresponds to the $\mathrm{SiO} v=1 J=1-0$ which is maser in nature in this O-rich evolved star. No spurious signal potentially produced by the plasma and the associated high-voltage electronics were detected. Only $150 \mathrm{MHz}$ of the $2 \mathrm{GHz}$ of instantaneous bandwidth of these observations are shown.

temperature at the back of the gas cell and a second integration using the sky as cold load. During most experiments the sky was clear and the antenna was pointing at 89 degrees elevation. The estimated cold load temperature was obtained for each scan from the weather conditions using the atmospheric transmission model (ATM; Cernicharo 1985; Pardo et al. 2001) and assuming a forward efficiency of $90 \%$ approximately. It was typically $40 \mathrm{~K}$ at $43 \mathrm{GHz}$. We also tested a cold load consisting of a mirror behind the gas cell pointing to an absorber inmersed in a liquid nitrogen tank. Results were worse than using the sky since we observed standing waves in the reference spectrum. The uncertainty on the calibration has been estimated to be less than $3 \%$, based on the repeatability of the receiver noise temperature after a significant number of measurements across the receiver bandwidth using the hot and cold loads just in front of the receiver beam. 
Most of the observations were performed using the frequency switching technique (FSW) with a maximum throw of $\pm 24 \mathrm{MHz}$ around the central frequency, one second integration per phase and $10 \mathrm{~ms}$ for the blank time. Each scan was integrated typically between one and three minutes. The calibration applied after doing the substraction between both phases was done at the central frequency giving a non flat baseline at the final spectra. In order to get a flatter baseline we used a procedure which consisted in doing a frequency switch cycle with the gas cell empty and the cold load, averaging 25 channels around each spectral channel and using that averaged reference spectrum to substract it from the calibrated result.

Some observations were done using the standard spatial ON-OFF technique; a typical scan consisted in three phases of $30 \mathrm{~s}$ of integration time with the gas cell empty, $60 \mathrm{~s}$ with the gas cell full and another $30 \mathrm{~s}$ with the gas cell empty. This technique resulted in flatter baselines but it was time consuming and was dependent on gain variations with time.

The frequency switch was used for static or flowing neutral gases while on-off techniques were also used when working with the radiofrequency generator that ionized the gas in the cell creating a plasma with the off phase corresponding to the plasma switched off. A blanking time of a few seconds was introduced between the on and off phases to allow pumping the by-products of the plasma phase.

To check that the radiofrequency generator did not inject RFI noise we performed several ON-OFF scans, $30 \mathrm{~s}$ integration time per phase of a $\mathrm{He}(0.3 \mathrm{mbar}) 60 \mathrm{~W}$ plasma being the on phase with the generator switched $\mathrm{ON}$ and the OFF phase with the generator switched off as during the experiments with other gases. System temperature and noise level were not affected by the RF generator. Additional tests consisting in observing an astronomical source, TX Cam, which exhibits a strong maser in the $J=1-0 v=1$ line of $\mathrm{SiO}$. The observations were performed before the installation of the cell, with the cell in the optical path of the receiver but with the plasma generator off, and finally with the plasma switched on. No spurious signal was detected over the $2 \mathrm{GHz}$ of instantaneous bandwidth of the spectrometer. Moreover, the intensity of the astronomical line was the same in the three cases ensuring the total transparency of the gas cell device to electromagnetic radiation coming from the sky (see Fig. 5).

The setup of the receiver, calibration and observation scans were managed by a software package developed by the authors and written in Python, which provides a command line interface. The software monitors the status of all devices, including the pressure sensors at the gas cell and the power of the radiofrequency oscillator when used. All parameters were stored in a database per scan for logging purposes. Data were recorded in ASCII files and converted on the fly into GILDAS compatible spectra and some status parameters were used to populate the headers of each scan. CLASS was later used for the analysis ${ }^{1}$.

\subsection{Spectral sensitivity}

The measurements have been performed by frequency switching (for long integrations), or by switching between the cell filled with the experimental gas and the cell completely empty (for fast data acquisition), or by swiching the plasma on and off (see above). The switching speed depends on the stability of the receivers. The acquistion data rate during the experiment can be as low as $0.1 \mathrm{~s}$ and depends on the chemical time of the experiments. For fast chemical experiments the OFF observations will

\footnotetext{
See, http://www.iram. fr/IRAMFR/GILDAS
}

last a few seconds and will be performed only at the beginning and at the end of the experiment. Hence, the noise of the observed spectra is dominated by the $\mathrm{ON}$ acquisition time, and is given by

$$
\sigma=\frac{T_{\mathrm{sys}}}{\sqrt{\Delta v t}},
$$

where $T_{\text {sys }}$ is the system temperature (in $\mathrm{K}$ ), $t$ is the integration time (in seconds), and $\Delta v$ is the spectral resolution (in $\mathrm{Hz}$ ). For a system temperature of $100 \mathrm{~K}, t=1 \mathrm{~s}$, and $\Delta v=0.035 \mathrm{MHz}$ the noise observed in the spectra is $0.54 \mathrm{~K}$. For optically thick lines the expected intensity is $230 \mathrm{~K}$ (see Sect. 3), and for optically thin lines it is $\sim 230 \times \tau$ (see Sect. 3). Hence, we expected to measure line opacities $\sim 10^{-2}$ at $5 \sigma$ in $1 \mathrm{~s}$ of observing time. We note that for optically thick transitions the system temperature to be considered for the calculation of the noise in the frequency range covered by the lines is $T_{\text {rec }}+T_{\text {cold }}+T_{\text {gas }}-T_{\text {cold }}=T_{\text {rec }}+T_{\text {gas }}$. For the frequencies and gas partial pressure of our experiments (see below) all observed lines are optically thin. Hence, the contribution of the lines to the spectral noise in their frequency ranges is negligible.

The sensitivity of thermal emission spectroscopy is lower than that of CHIRP pulse spectrometers at low frequency. For frequency observations in the millimeter and submillimeter domain the very large instantaneous bandwidth provided by low noise cooled radio astronomical receivers offers a sensitivity comparable, and even better, than that provided by standard laboratory absorption measurements using warm detectors and narrow frequency bands.

\subsection{Selecting appropriated windows for the gas cell}

Two types of windows were initially considered for the gas cell: a) thick $(9.60 \mathrm{~mm})$ fused quartz $\left(\epsilon_{r}=3.8\right)$ and b) thin $(75 \mu \mathrm{m})$ Upilex polyimide film $\left(\epsilon_{r}=3.3\right)$. The fused quartz windows were initially selected for their compatibility with ultra-high vacuum systems, their robustness and their chemical inertness, but were expected to reflect a significant part of the incoming radiation at some frequencies. On the other hand, Upilex windows with low dielectric constant and small thickness provide an almost ideal transmission in the frequency range of interest, but are very fragile and prone to degradation by mechanical stress and chemical reactions at their surfaces.

In order to gain a better understanding of the impact of the electrical properties of the windows on the sensitivity of the experiment, a simple model of the combination of the gas cell and the cryogenic receiver was elaborated. The windows were simulated as transmission lines with characteristic impedances and electrical lengths according to their dielectric constant and thickness. The receiver was assumed to present a flat noise temperature of $50 \mathrm{~K}$ in the entire band. As the input and output windows are tilted by approximately $11^{\circ}$ with respect to the optical axis, it was assumed that no interference exist between them. However, the interference between the two faces of each window and the reflection of the ambient thermal radiation towards the receiver were included. Figure 6 presents the comparison of the simple model prediction with the experimental results of the receiver noise temperature obtained by measurement with hot-cold load at the input of the gas cell. We note the severe degradation of the sensitivity around $44 \mathrm{GHz}$ in the case of the fused quartz windows.

The effect of dissipative loss in dielectric materials $(\tan \delta$ ) is not obvious from the plot shown in Fig. 6 and it is relatively 


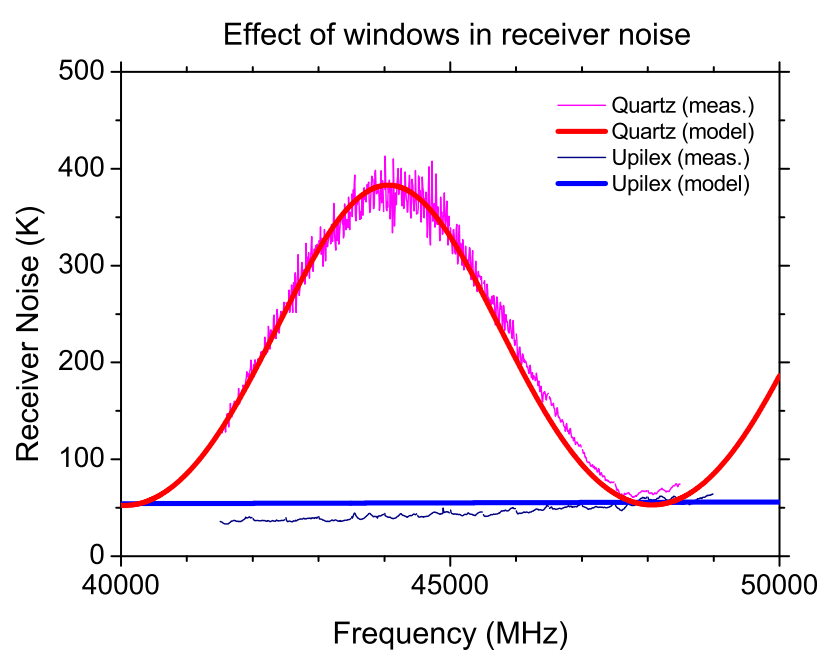

Fig. 6. Effect on the system temperature of the windows used in the experiment. The receiver temperature was measured between 41.5 and $49 \mathrm{GHz}$. The quartz windows, which are best suited for high vacuum, have a poor performance and introduce a significant loss of sensitivity. The Upilex windows have a very good behaviour concerning transmission but are much more sensitive to stress when the chamber is evacuated. The thick lines correspond to the predicted receiver noise temperature by the model described in the text.

small in the two cases. Amorphous quartz has very low losses ( $\tan \delta \simeq 0.0001$ ), but Upilex is slightly worse than other similar polymer materials normally used as microwave dielectrics ( $\tan \delta \simeq 0.005$ ). To illustrate the effect of dissipative loss in the two materials we can compare the estimated added noise due to dielectric loss for the two windows for quartz and Upilex at $48 \mathrm{GHz}$ (coincident with the minimum reflection of quartz). It is found to be $0.80 \mathrm{~K}$ for Upilex and $1.56 \mathrm{~K}$ for quartz.

Despite the practical problems posed by the thin Upilex windows exposed above, most of the experiments were performed with them due to their good electrical properties and superior sensitivity in the frequency range of interest. After the completion of the experiments shown in this paper Teflon windows were used, fitted with quarter wave matching layers on both sides in in order to decrease their reflectivity. The total thickness was $7 \mathrm{~mm}$. The matching layers were machined as concentric grooves of $1.4 \mathrm{~mm}$ depth and $1.5 \mathrm{~mm}$ width spaced by $1.5 \mathrm{~mm}$. The reflection loss of the windows was expected to be below $-30 \mathrm{~dB}$ in the whole band of the receiver. The noise contribution due to dielectric dissipative loss has been measured and found to be negligible. Teflon offers superior safety conditions than Upilex and similar transmission performances.

\section{Absolute data calibration}

Let us assume that the windows of the cell are transparent at microwave wavelengths. The receivers are equipped with HEMTs (see below) so that the data are in SSB mode and we do not have to deal with the always uncertain gains of upper and lower sidebands typical in SIS superheterodyne receivers. Facing the receiver, behind the cell, there is a black body at temperature $T_{\mathrm{c}}$ (cold load, see Fig. 1). The gas in the cell is at temperature $T_{\mathrm{g}}$ with a volume density high enough to maintain it under thermal equilibrium conditions, that is, all rotational levels are populated following a Boltzmann distribution at a single rotational temperature which is identical to the kinetic temperature of the gas. As frequencies in our experiment are below $50 \mathrm{GHz}$ and the physical temperature of the cell is $\sim 300 \mathrm{~K}$, it turns out that $h v / k_{\mathrm{B}} T<0.01$ ( $h$ and $k_{\mathrm{B}}$ are the Planck and Boltzmann constants respectively). In those conditions, we can assume the Rayleigh-Jeans approximation applies to the line flux $I_{v}$ and use a brightness temperature, $T_{\mathrm{B}}$, so that $I_{v}(T)=2 v^{2} k_{\mathrm{B}} T_{\mathrm{B}} / c^{2}$. Hence, the line intensity can be given as a temperature so that the signal detected by a receiver in front of the cell at a frequency $v$ (assumed to be close to the central frequency $v_{\mathrm{ul}}$ of the molecular transition $\mathrm{u} \rightarrow \mathrm{l}$ ) can be written as

$T_{\mathrm{B}}^{\mathrm{ON}}(v)=T_{\mathrm{c}} \mathrm{e}^{-\tau\left(v, T_{\mathrm{g}}\right)}+T_{\mathrm{g}}\left(1-\mathrm{e}^{-\tau\left(v, T_{\mathrm{g}}\right)}\right)=\left(T_{\mathrm{c}}-T_{\mathrm{g}}\right) \mathrm{e}^{-\tau\left(v, T_{\mathrm{g}}\right)}+T_{\mathrm{g}}$,

where $\tau\left(v, T_{\mathrm{g}}\right)$ is the gas opacity integrated along the cell length at frequency $v$ and temperature $T_{\mathrm{g}}$ (see below). When $T_{\mathrm{c}}=T_{\mathrm{g}}$, there is a lack of contrast and therefore the signal detected will not display any spectral signature: $T_{\mathrm{B}}(v)=T_{\mathrm{g}}$. In this consideration we have neglected the emissivity and absorption of the cell windows (see below). The opacity of the line depends on the dipole moment of the molecule, the total number of molecules along the path, the frequency, and the temperature (through the partition function) and will be discussed in the next section. When the receiver observes through an empty cell the detected signal will be

$T_{\mathrm{B}}^{\mathrm{OFF}}(v)=T_{\mathrm{c}}$.

The signal $T_{\mathrm{B}}^{\mathrm{ON}-\mathrm{OFF}}(v)$, obtained by the standard ON-OFF technique in radioastronomy will be given by

$T_{\mathrm{B}}^{\mathrm{ON}-\mathrm{OFF}}(v)=\left(T_{\mathrm{g}}-T_{\mathrm{c}}\right)\left(1-\mathrm{e}^{-\tau\left(v, T_{\mathrm{g}}\right)}\right)$.

If $T_{\mathrm{g}}>T_{\mathrm{c}}$ we will observe the spectral lines in emission. If $T_{\mathrm{g}}<T_{\mathrm{c}}$ they will be in absorption. For practical purposes we can assume $T_{\mathrm{c}}=70 \mathrm{~K}$ and $T_{\mathrm{g}}=300 \mathrm{~K}$. In the optically thick case, that is, $\tau \gg 1, T_{\mathrm{B}}^{\mathrm{ON}-\mathrm{OFF}}=230 \mathrm{~K}$. In the optically thin case, $\tau \ll 1, T_{\mathrm{B}}^{\mathrm{ON}-\mathrm{OFF}}=230 \tau\left(v, T_{\mathrm{g}}\right) \mathrm{K}$. In the above equations we have assumed that air is practically transparent at the wavelengths of the experiment. However, $\mathrm{O}_{2}$ has a cluster of absorption lines in the range $50-70 \mathrm{GHz}$ and another one centred at 118.75 GHz. Water has an absorption feature at $22 \mathrm{GHz}$. Hence, the air path in this experiment should be maintained as short as possible. Nevertheless, the absorption produced by the air can be corrected using the ATM model (Cernicharo 1985; Pardo et al. 2001).

A more tricky problem is the absorption by the material used for the windows of the cell. As, in general, that absorption will not be negligible, we can generalize Eq. (2) as follows:

$$
\begin{aligned}
T_{\mathrm{B}}^{\mathrm{ON}}(v)= & {\left[\left\{T_{\mathrm{c}} \mathrm{e}^{-\kappa_{v}}+T_{\mathrm{w}}\left(1-\mathrm{e}^{-\kappa_{\nu}}\right)\right\} \mathrm{e}^{-\tau\left(v, T_{\mathrm{g}}\right)}\right.} \\
& \left.+T_{\mathrm{g}}\left(1-\mathrm{e}^{-\tau\left(v, T_{\mathrm{g}}\right)}\right)\right] \mathrm{e}^{-\kappa_{v}}+T_{\mathrm{w}}\left(1-\mathrm{e}^{-\kappa_{v}}\right),
\end{aligned}
$$

where $T_{\mathrm{w}}$ is the temperature of the windows (in principle identical to $T_{\mathrm{g}}$ ), and $\kappa_{v}$ is the opacity of the windows at frequency $v$. The first term represents the emission of the cold load attenuated by the first window. The second term represents the emission of the first window. Both terms are attenuated by the gas in the cell. The third term represents the emission of the gas. Finally the window in front of the receiver will attenuate all the previous terms and emit itself (last term). In case of an empty cell, we get the OFF signal:

$T_{\mathrm{B}}^{\mathrm{OFF}}(v)=\left[T_{\mathrm{c}} \mathrm{e}^{-\kappa_{v}}+T_{\mathrm{w}}\left(1-\mathrm{e}^{-\kappa_{v}}\right)\right] \mathrm{e}^{-\kappa_{v}}+T_{\mathrm{w}}\left(1-\mathrm{e}^{-\kappa_{v}}\right)$,

and the detected ON-OFF signal will be

$T_{\mathrm{B}}^{\mathrm{ON}-\mathrm{OFF}}(v)=\left[T_{\mathrm{g}}-T_{\mathrm{c}} \mathrm{e}^{-\kappa_{v}}-T_{\mathrm{w}}\left(1-\mathrm{e}^{-\kappa_{v}}\right)\right]\left(1-\mathrm{e}^{-\tau\left(v, T_{\mathrm{g}}\right)}\right) \mathrm{e}^{-\kappa_{v}}$. 
The absolute calibration is obtained, as mentioned in Sect. 2.2, through the use of two microwave absorbers at different temperatures (liquid $\mathrm{N}_{2}$, or clear sky, and ambient). The detected flux, $W$, is transformed into temperature, $T$, through

$T=W\left(T_{\mathrm{h}}-T_{\mathrm{c}}\right) /\left(W_{\mathrm{h}}-W_{\mathrm{c}}\right)$,

where $W_{\mathrm{h}}$ and $W_{\mathrm{c}}$ are the signals detected by the receiver from the hot and cold loads, $W$ is the flux detected in a given observation phase (ON, OFF, or FSW [frequency switching]), and $T_{\mathrm{h}}$ and $T_{\mathrm{c}}$ are the temperatures of the hot and cold loads.

The accuracy of the calibration can be degraded by the atmosphere, the uncertainty in the transmission of the cell windows, as well as instrumental baseline effects. The short atmospheric path involved in the experiment ensures that the atmosphere is not perturbing the observations. Taking this factor into account, we can estimate uncertainties below $5 \%$ in our measurements (to be compared with the $3 \%$ from the repeatability obtained in the measurement of the receiver noise temperature). Hence, the partial pressures, or molecular abundances, of the gases in the cell derived from the observed intensities of their rotational lines will exhibit similar error bars $(\sim \pm 5 \%)$.

As the observation frequency increases, the Rayleigh-Jeans approximation starts to fail. Consequently, in the submillimeter and far-infrared domains $I_{v}$ has to be replaced in the calibration equations by the full Planck function, $B_{v}(T)$, given by

$B_{v}(T)=\left(2 h v^{3} / c^{2}\right)\left(\mathrm{e}^{\left(h v / k_{\mathrm{B}} T\right)}-1\right)^{-1}$,

where $v$ is the frequency, $h$ is the Planck constant, $c$ is the speed of light, and $k_{\mathrm{B}}$ is the Boltzmann constant.

\section{Molecular thermal emission in the gas cell}

The volume density, $n$, of a given gas in molecules $\mathrm{cm}^{-3}$ (hereafter these units are referred to as $\mathrm{cm}^{-3}$ ) in the cell is given by

$n=7.2431 \times 10^{18} P / T$,

where $P$ is the partial pressure (in mbar) and $T$ the kinetic temperature (in $\mathrm{K}$ ) of the gas. The gas column density, $N$, in $\mathrm{cm}^{-2}$ along the optical path $d$ (in $\mathrm{cm}$ ) of the experiment is given by

$N=7.2431 \times 10^{18} d P / T$.

The opacity of a molecular line corresponding to a transition $\mathrm{u} \rightarrow \mathrm{l}$ is given by

$\tau\left(v, T_{\mathrm{g}}\right)=c^{2} N g_{\mathrm{u}} /\left(8 \pi Z v_{\mathrm{ul}}^{2}\right) \times \mathrm{e}^{-E_{\mathrm{l}} / T_{\mathrm{g}}} A_{\mathrm{ul}}\left(1-\mathrm{e}^{-h v_{\mathrm{ul}} / k_{\mathrm{B}} T_{\mathrm{g}}}\right) \phi(v)$,

where $Z$ is the partition function, $A_{\mathrm{ul}}$ is the Einstein coefficient of the transition, $g_{\mathrm{u}}$ is the degeneracy of the upper level, $E_{1}$ the energy of the lower level of the transition (in K), and $\phi(v)$ is the normalized line profile. The Einstein coefficient is given by

$A_{\mathrm{ul}}=\frac{64 \pi^{4}}{3 g_{\mathrm{u}} h c^{3}} v_{\mathrm{ul}}^{3} \mu_{\mathrm{ul}}^{2}$,

where $\mu_{\mathrm{ul}}^{2}$ is the square of the transition dipole moment which, in turn, can be written as $S_{\mathrm{ul}} \mu^{2}$, where $S_{\mathrm{ul}}$ is the so-called transition line strength and $\mu$ is the permanent dipole moment of the molecule. Therefore, the line opacity can be written as follows:

$$
\begin{aligned}
\tau\left(v, T_{\mathrm{g}}\right) & =\left(8 v_{\mathrm{ul}} \pi^{3} / 3 h c\right)\left(N S_{\mathrm{ul}} \mu^{2} / Z\right)\left(\mathrm{e}^{-E_{1} / T}-\mathrm{e}^{-E_{\mathrm{u}} / T}\right) \phi(v) \\
& =I_{\mathrm{ul}}(T) N \phi(v) .
\end{aligned}
$$

The parameter $I_{\mathrm{ul}}$ is provided for a significant number of molecules in the JPL (Pickett et al. 1998) and CDMS (Müller et al. 2005) line catalogues. The MADEX code (Cernicharo 2012) also provides this value for more than 5600 molecules when used in its LABO mode.

The thermal or Doppler $\left(\Delta \nu_{\mathrm{D}}\right)$ line width (half width at half maximum, HWHM), for a molecule of mass $M$ (in atomic units) is given by

$\Delta v_{\mathrm{D}}=3.581 \times 10^{-7} v_{\mathrm{ul}}(T / M)^{1 / 2}$.

The Doppler line profile can be assumed to be a Gaussian with a HWHM of $\Delta v_{\mathrm{D}}$. The spectral signal will be maximum at the line centre (corresponding to the maximum absorption coefficient per unit length, $\alpha_{\max } ; \tau_{\max }=\alpha_{\max } \times d$ ). In the so-called Doppler limit it is given by

$\tau_{\max }(T)=I_{\mathrm{ul}}(T) \frac{T_{0}}{T} \frac{P}{\Delta v_{\mathrm{D}}} \times 151.194 \times d$,

where $I_{\mathrm{ul}}(T)$ is in units of $\mathrm{nm}^{2} \mathrm{MHz}, P$ is the partial pressure of the gas in Torr, $\Delta v_{\mathrm{D}}$ is the Doppler HWHM in MHz, and $d$ the length of the cell in $\mathrm{cm}$, and $T_{0}=300 \mathrm{~K}$.

Pressure or collisional broadening will induce line profile with a HWHM given by $\Delta v_{\mathrm{C}}=\Delta v_{\mathrm{C}}^{0} P$, where $P$ is the pressure and $\Delta v_{\mathrm{C}}^{0}$ is the broadening induced at a reference pressure (normally 1 Torr; 1 Torr $=1.33322$ mbar).

Above a certain pressure the line profile will be dominated by the effect of collisions on the width of the energy levels involved in the transition. The profile $\phi(v)$ of a collisionally broadened line can be approximated by the Van Vleck-Weisskopf (VVK) profile (see Appendix A). When the line is collisionally broadened the absorption coefficient $\tau_{\max }$ does not depend on the pressure as both, the broadening and the number of particles have the same dependence with the pressure. The value of $\tau_{\max }(T)$ at temperature $T$ is given by:

$\tau_{\max }(T)=I_{\mathrm{ul}}(T) \frac{T_{0}}{T \Delta v_{\mathrm{C}}^{0}} \times 102.458 \times d$.

In this expression $\Delta v_{\mathrm{C}}^{0}$ is in $\mathrm{MHz} /$ Torr. If $\Delta v_{\mathrm{C}}^{0}$ is given in $\mathrm{MHz} / \mathrm{mbar}$ then

$\tau_{\max }(T)=I_{\mathrm{ul}}(T) \frac{T_{0}}{T \Delta v_{\mathrm{C}}^{0}} \times 76.850 \times d$,

(Eqs. (15)-(18) are taken or adapted from Pickett et al. 1998). To illustrate this, we show in Fig. 7 the expected brightness temperature of the CS $J=1-0$ line for a cell length of $40 \mathrm{~cm}$, ambient temperature of $300 \mathrm{~K}$, and cold load temperature of $70 \mathrm{~K}$. Two cases have been considered, the cell filled only with CS $\left(P_{\mathrm{T}}=P_{\mathrm{CS}}\right)$ and the cell filled with CS and another gas $\left(\mathrm{N}_{2}\right)$ with a total pressure, $P_{\mathrm{CS}}+P_{\mathrm{N}_{2}}$, of 0.3 mbar. Brightness temperatures for CS $J=1-0$ can be obtained using $I_{1-0}(300 \mathrm{~K})=$ $2.381 \times 10^{-4} \mathrm{~nm}^{2} \mathrm{MHz}$ (Müller et al. 2005), and Eq. (7). In the case $P_{\mathrm{T}}=P_{\mathrm{CS}}=0.3 \mathrm{mbar}$, the volume density of CS is $7.22 \times 10^{15} \mathrm{~cm}^{-3}$, and the corresponding column density in the cell is $2.89 \times 10^{17} \mathrm{~cm}^{-2}$. The HWHM of CS has been assumed to be $7.5 \mathrm{MHz}$ at 1 Torr for CS-CS self broadening and $\mathrm{CS}-\mathrm{N}_{2}$ broadening. Hence, for the case of constant total pressure of 0.3 mbar the linewidth of the observed transition should be 3.38 MHz. The maximum brightness temperature that we could expect in the case $P_{\mathrm{T}}=P_{\mathrm{CS}}=0.3 \mathrm{mbar}$ for this CS line is $27.3 \mathrm{~K}$ and will be obtained for a CS partial pressure of $>0.05$ mbar. In the case that the partial pressure of CS is low compared with the 


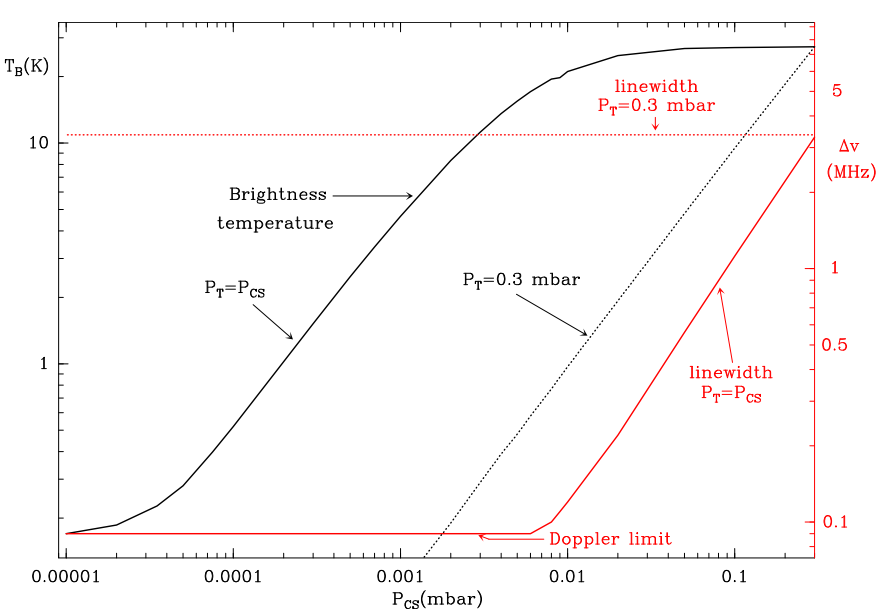

Fig. 7. Expected brightness temperature (black lines) and linewidth (red lines) for the CS $J=1-0$ line for two different cases. The continuous lines correspond to the cell filled only with CS and $P_{\mathrm{T}}=P_{\mathrm{CS}}$, while the dashed lines correspond to a cell filled with $\mathrm{N}_{2}$ and CS at a total constant pressure of $0.3 \mathrm{mbar}$. The $x$-axis corresponds to the partial pressure of CS.

total pressure (dashed lines) the width of the transition will be always nearly the same, that is, $3.38 \mathrm{MHz}$. However, the intensity varies linearly with pressure from $27.3 \mathrm{~K}$ for $P_{\mathrm{CS}}=0.3 \mathrm{mbar}$ and $P_{\mathrm{N}_{2}}=0$, to $0.2 \mathrm{~K}$ for $P_{\mathrm{CS}} \simeq 0.007 \mathrm{mbar}$ and $P_{\mathrm{N}_{2}}=0.293 \mathrm{mbar}$.

It must be noted that $\Delta v_{\mathrm{C}}^{0}$ also depends on the temperature, through a relation usually expressed as

$\Delta v_{\mathrm{C}}^{0}(T)=\Delta v_{\mathrm{C}}^{0}\left(T_{0}\right) \times\left(\frac{T_{0}}{T}\right)^{n}$,

where $T_{0}$ is a reference temperature, $n$ is determined empirically for each molecule, being typically $n \simeq 0.7-1$. See Appendix A for more details on the line profile and the effect of broadening on the intensity.

\section{Results}

\subsection{Static or flowing gas}

In the first experiments we selected ethanol and methanol at different pressures as test molecules, because of their many lines in the $Q$ band. The temperature inside the gas cell was the ambient temperature in the receiver room of the telescope and close to $300 \mathrm{~K}$. However, these molecules have large partition functions and self-broadening coefficients at $300 \mathrm{~K}$, both factors playing against the peak intensity of the spectral lines we could detect. Methanol has two symmetry species, A and E, and ethanol two conformers. Moreover, at $300 \mathrm{~K}$ several vibrational and internal rotational modes are populated, also decreasing the expected intensities of the rotational lines at their ground states. For example, for $\mathrm{CH}_{3} \mathrm{OH}$, the expected peak intensity of its line at $44069 \mathrm{MHz} 7_{0,7}-6_{1,6} A^{+}$transition, $I(300 \mathrm{~K})=8.801 \times$ $10^{-7} \mathrm{~nm}^{2} \mathrm{MHz}$ ) calculated by MADEX (Cernicharo 2012) is only $0.3 \mathrm{~K}$. For the $46980 \mathrm{MHz}$ ethanol line $\left(7_{1,6}-7_{0,7}\right.$ transition of the trans conformer, $\left.I(300 \mathrm{~K})=3.352 \times 10^{-6} \mathrm{~nm}^{2} \mathrm{MHz}\right)$, the expected peak intensity is $0.2 \mathrm{~K}$ for the conditions of the experiment. The first experimental run was held in October 2015 using the quartz windows. To obtain the spectra we performed ON/OFF scans, according to Eqs. (4) and (7), with ON: chamber filled with the gas, and OFF: empty chamber. However, no lines were observed.

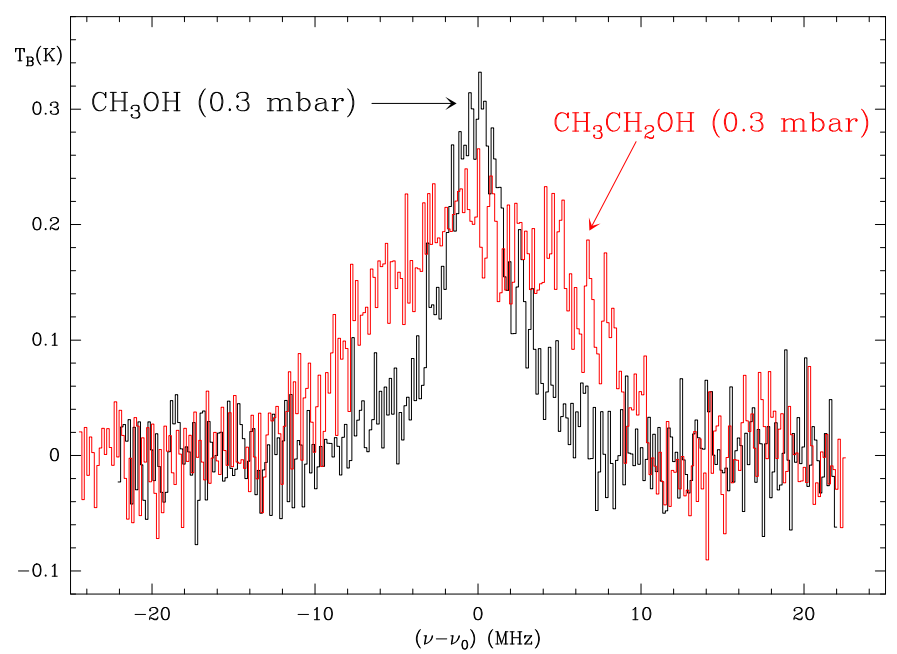

Fig. 8. Observed lines of methanol and ethanol at 44069 and $46980 \mathrm{MHz}$, respectively.

The problem arose from the quartz windows which reduce the sensitivity (see Fig. 6) and, in addition, introduce baseline artefacts due to internal reflections. We have also noticed that thermal emission from people around the chamber leads to significant degradation of the baseline. In the following experiments we installed the Upilex windows which have a much better transmission (see Sect. 2.4). Figure 8 shows the lines of $\mathrm{CH}_{3} \mathrm{OH}$ and $\mathrm{CH}_{3} \mathrm{CH}_{2} \mathrm{OH}$ after 10 min of $\mathrm{ON}-\mathrm{OFF}$ observing time at a total static pressure of 0.3 mbar when the chamber was filled with each of these gases. From just a single measurement of the ethanol line we could estimate a broadening coefficient of $20 \mathrm{MHz} / \mathrm{mbar}$. Figure 9 shows the methanol line observed at different pressures (baseline ripples can be seen in the $0.1 \mathrm{mbar}$ spectrum). As pointed out in the previous section, in the pressure broadened regime, the peak height remains constant while the linewidth changes with pressure. For pressures below $0.02 \mathrm{mbar}$ the line reaches its Doppler width (see Fig. 10). Typical integration times were $10 \mathrm{~min}$ during these measurements except for the lowest pressure for which we integrated during $40 \mathrm{~min}$. All spectra were fitted to Voigt profiles, with the Doppler width fixed to the expected value at $300 \mathrm{~K}(97 \mathrm{kHz})$, and peak intensities, collisional linewidths (FWHM) and integrated intensities where derived from the fit. Figure 10 shows these experimental values versus pressure, and the linear fits that show the linear dependence of collisional width and integrated intensity with pressure. The self-broadening coefficient thus derived is $13.2 \pm 0.6 \mathrm{MHz} / \mathrm{mbar}$ (HWHM). The upper panel of Fig. 10 also shows the expected evolution of the peak intensity using the derived broadening parameter.

The Upilex windows were adopted for all the other observing periods. In the second observing run in April 2016 we used $\mathrm{HCOOH}$ and OCS as probe molecules for static or flowing conditions. The observed baselines were very good for standard ON/OFF observations but exhibited still strong baseline ripples in FSW mode. However, we noticed that the rippled instrumental baseline was constant in time when the sky was used as cold load. Hence, an instrumental baseline was derived before filled-cell scans by integrating with the empty cell for $40 \mathrm{~s}$ in FSW mode. This curve was then subtracted to all subsequent observations resulting in very flat baselines.

The partition function of OCS is smaller than that of methanol and ethanol at $300 \mathrm{~K}$. For $\mathrm{HCOOH}$ (formic acid) the partition function is slightly smaller than that of methanol and 
I. Tanarro et al.: A gas cell for astrochemical simulations

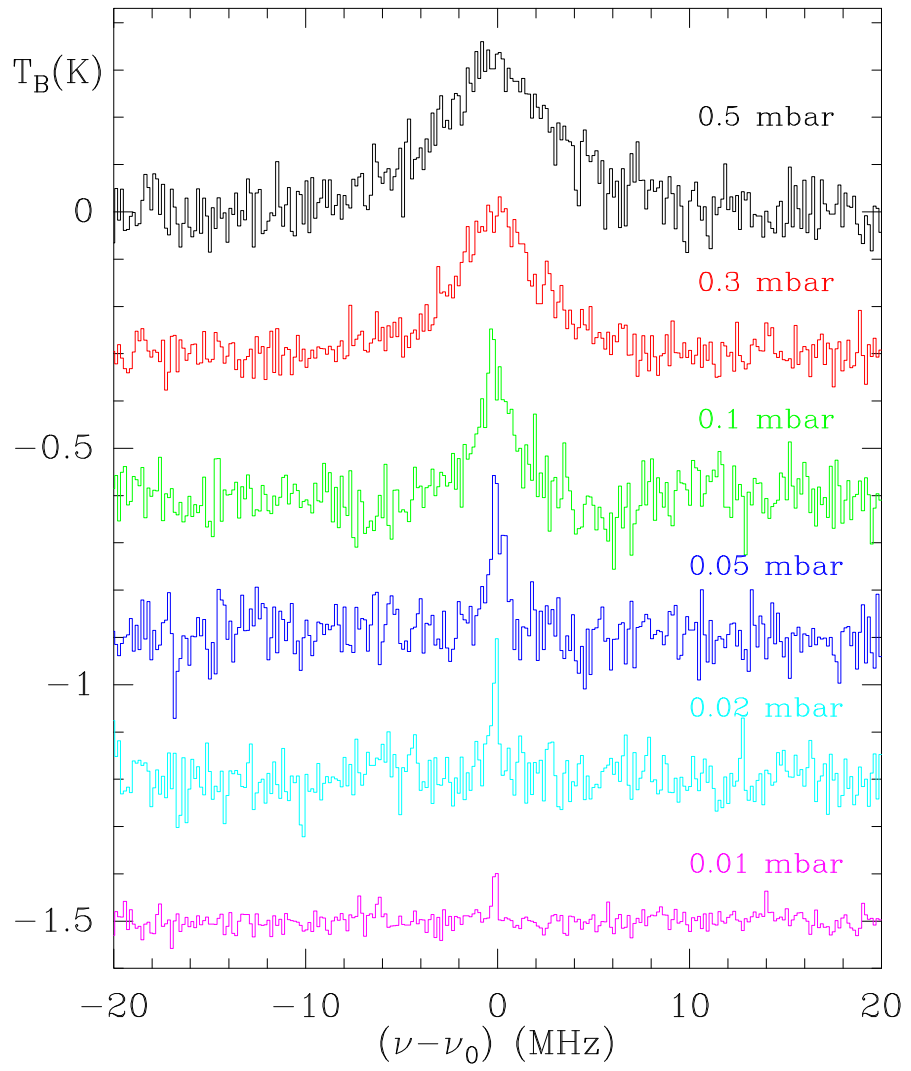

Fig. 9. Observed line of methanol at $44069 \mathrm{MHz}\left(7_{0,7}-6_{1,6} A^{+}\right.$transition) for different values of the total gas pressure. The integration time was $40 \mathrm{~min}$ for the narrower feature. The spectral resolution is $180 \mathrm{kHz}$ obtained by a five channel smoothing of the original data. A shift by $-0.3 \mathrm{~K}$ is introduced in the vertical scale of each spectrum with respect to the previous one, as pressure decreases.

the expected intensities in the $41-49 \mathrm{GHz}$ range are of the same order than those of methanol and ethanol. Figure 11 shows two lines of the trans conformer of formic acid observed simultaneously. The observing time in these observations was $4 \mathrm{~min}$.

Figure 12 shows the observed line profile of OCS $J=4-3$ for different experimental conditions. The bottom spectrum corresponds to the plasma experiment and will be discussed below. Total observing time is around $10 \mathrm{~min}$ and the spectra have been smoothed to a spectral resolution of $1.0 \mathrm{MHz}$. The spectra show the rotational emission from OCS in the ground state and in its $v_{2}$ mode, placed at $749 \mathrm{~K}$ above the ground state. The observed intensities are those expected for a gas at $300 \mathrm{~K}$. During the experiment with a cell pressure of 0.5 mbar it was possible to detect also the $J=4-3$ transition of the $\mathrm{OC}^{34} \mathrm{~S}$ isotopologue (see insert in Fig. 12). From the observed spectra in Fig. 12 we derive $\Delta v_{\mathrm{C}}$ (HWHM), of $6.0 \pm 0.5 \mathrm{MHz} / \mathrm{mbar}$, in good agreement with Koshelev \& Tetryakov (2009).

When the gas cell is filled with OCS at a pressure of 0.3 mbar, and adopting the pressure broadening coefficient derived above, $T_{\mathrm{c}}=70 \mathrm{~K}$, and $T_{\mathrm{g}}=300 \mathrm{~K}$, then the MADEX code predicts an intensity for the OCS $J=4-3$ transition at $48651 \mathrm{MHz}$ of $\sim 3.3 \mathrm{~K}$ (see Appendix A). The observed OCS intensities, as shown in Fig. 12, are in agreement with these predictions. Hence, the partial pressure of the gases in the cell can be derived with high accuracy from the measurement of the thermally populated rotational lines. As expected for these volume densities no differences were found between the static and flowing gas case for identical pressures of the gas in the cell.

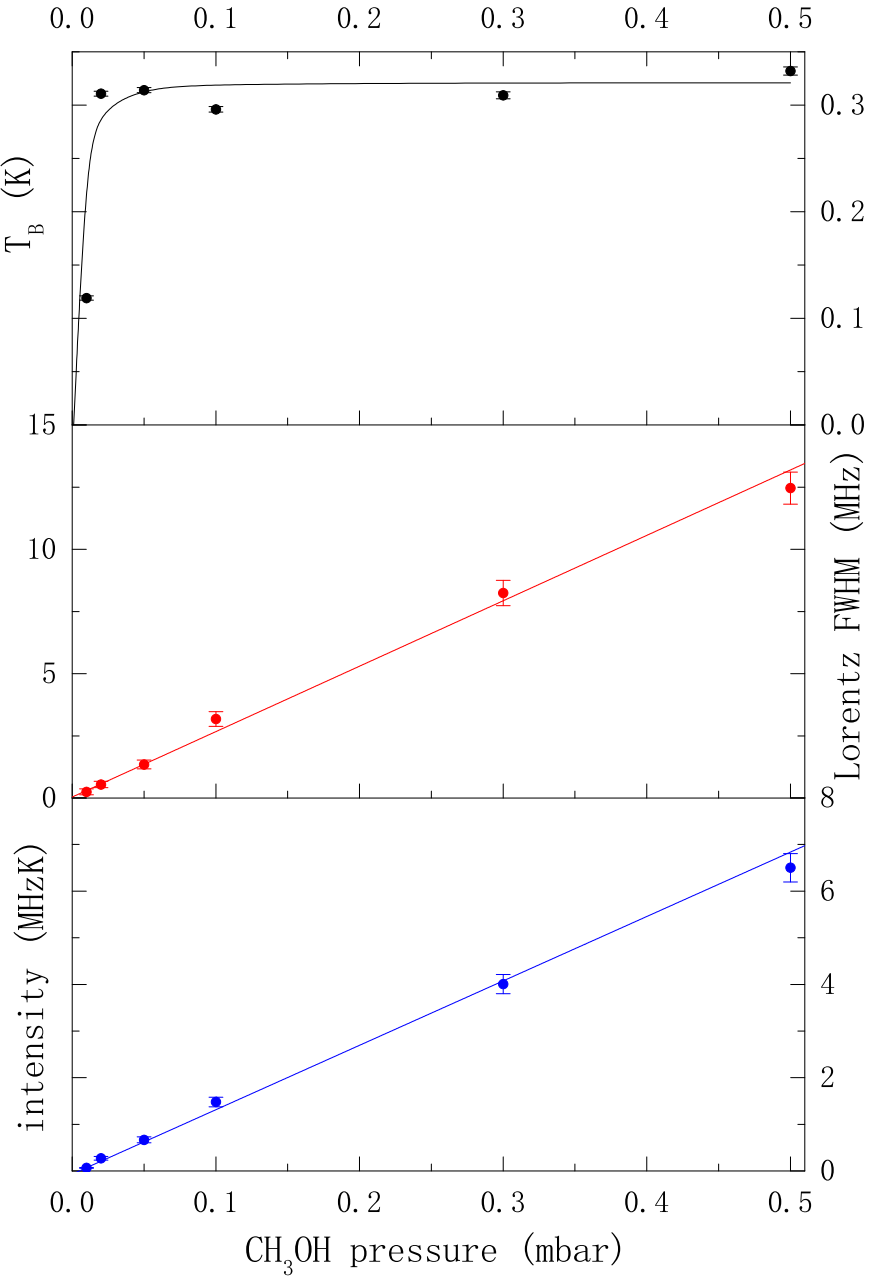

Fig. 10. Symbols (from top to bottom): peak intensity, collisional linewidth and integrated intensity derived from Voigt fits to the observed methanol line $\left(7_{0,7}-6_{1,6}, \mathrm{~A}^{+}\right)$; data from Fig. 9. In all fits the Gaussian width was fixed to the calculated value. Solid lines (from top to bottom): calculated peak intensity with the calculated broadening coefficient, and intensity, best fit of the collisional widths vs. pressure (the slope yields a broadening coefficient $\Delta v_{\mathrm{C}}=13.2 \pm 0.6 \mathrm{MHz} / \mathrm{mbar}$ ) and best fit of the integrated line intensity vs. pressure.

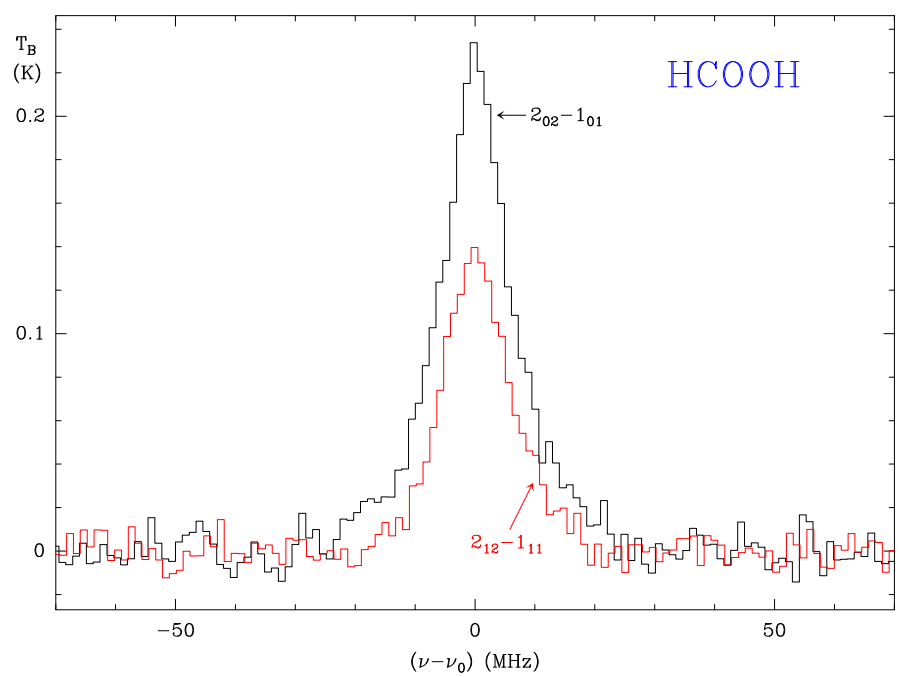

Fig. 11. Observed emission of the $2_{12}-1_{01}$ and $2_{02}-1_{01}$ trans $\mathrm{HCOOH}$ transitions at 43303 and $44911 \mathrm{MHz}$ respectively. The integration time was $4 \mathrm{~min}$. Both lines were observed simultaneously. The spectral resolution is $540 \mathrm{kHz}$ after a channel smoothing of the original data. 


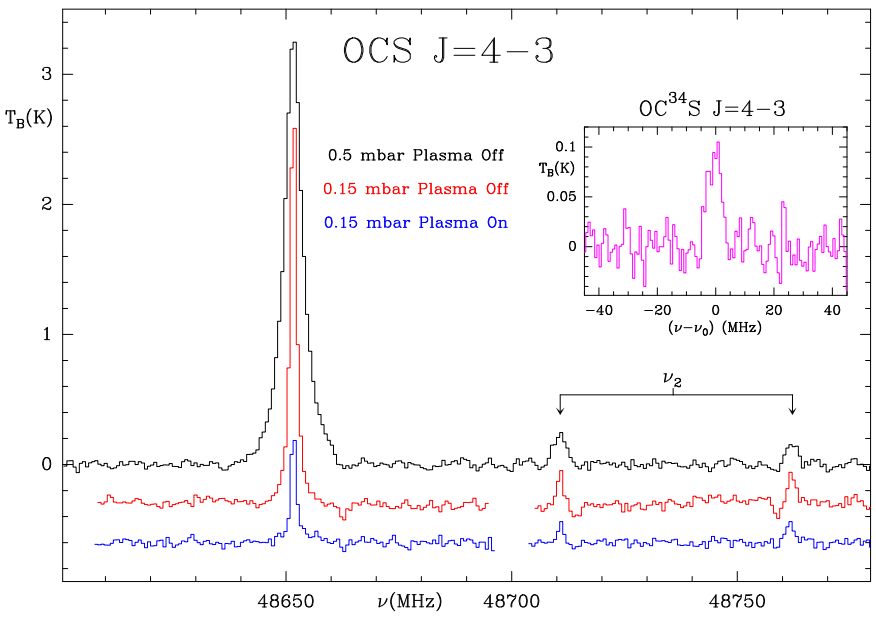

Fig. 12. Observed emission of the $J=4-3$ OCS rotational line for different experimental conditions. The doublet of the $v_{2} J=4-3$ transition is also detected in the experiments. The top spectrum was taken in ON/OFF mode, that is, signal through OCS filled cell minus signal through empty gas cell. The other spectra were acquired in FSW mode. The blanked channels in the baseline correspond to the negative features produced by the frequency folding of the spectra. The insert shows the $J=4-3$ line of $\mathrm{OC}^{34} \mathrm{~S}$ at $47462.353 \mathrm{MHz}$ for $P(\mathrm{OCS})=0.5 \mathrm{mbar}$.

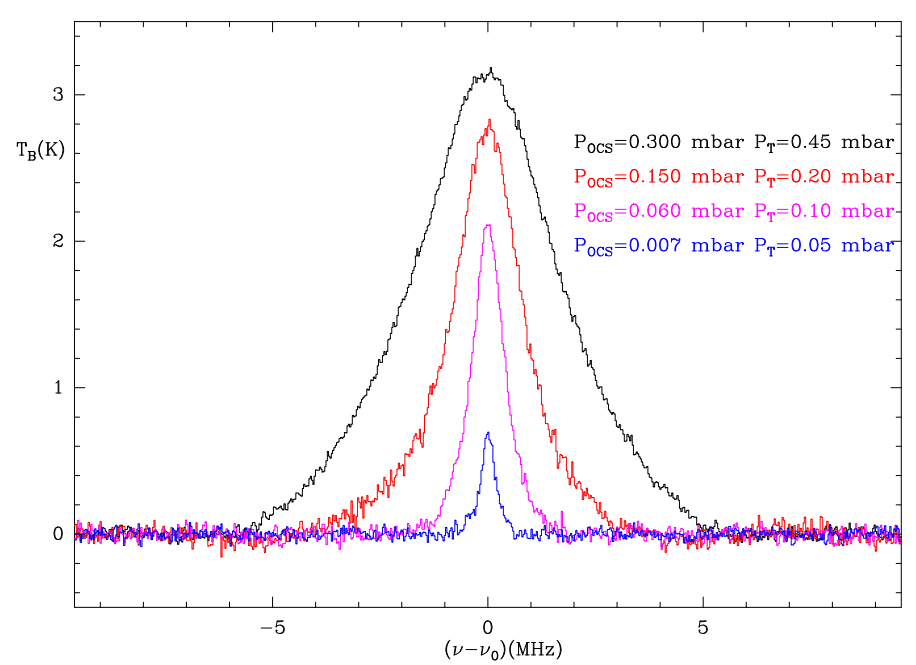

Fig. 13. Observed $J=4-3$ OCS transition at $48651.604 \mathrm{MHz}$ for different values of OCS and total pressures. The integration time was 4 min for the lowest pressure. The spectral resolution is $30 \mathrm{kHz}$.

Observations of the $J=4-3$ line of OCS at different partial and total pressures (in static conditions, with varying contributions of background air) are shown in Fig. 13. The mixing of OCS and air during this experiment was not well under control. Hence, we estimate that the total and partial pressures shown in Fig. 13 have uncertainties of $\sim 30 \%$.

\subsection{Cold plasma conditions}

The prototype of the gas cell was equipped with the cold plasma generation described in Sect. 2 (see Figs. 2-4). In this type of glow inductively coupled plasma (ICP) discharges the typical electron densities are $10^{9}-10^{10} \mathrm{~cm}^{-3}$, while typical electron temperatures are 3-5 eV (assuming Maxwellian distributions). The most energetic electrons, with energies spanning up to several

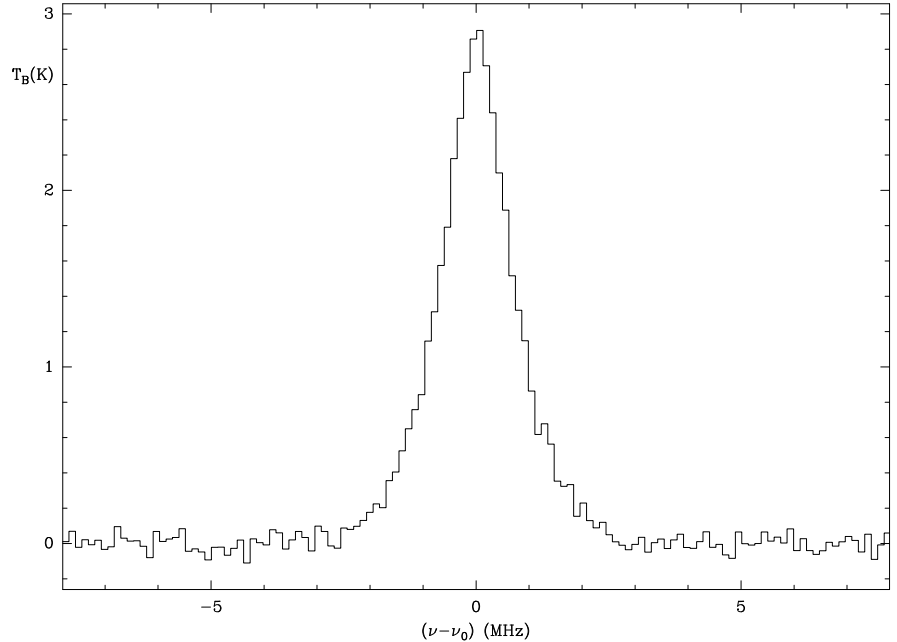

Fig. 14. Observed CS $J=1-0$ signal (centred at $48990.957 \mathrm{MHz}$ ) at $0.15 \mathrm{mbar}$ and $50 \mathrm{~W}$ OCS plasma. The integration time was $2 \mathrm{~min}$. The spectral resolution is $160 \mathrm{kHz}$.

tens of $\mathrm{eV}$, are responsible for the primary electron impact dissociation and ionization processes that begin the rich chemistry at low temperatures associated to this kind of plasmas.

First studies on the kinetics of cold OCS and $\mathrm{CS}_{2}$ plasmas produced at low pressure were carried out in 1980s by different authors. Clark \& De Lucia (1981) employed millimeter and submillimeter rotational spectroscopy, based on klystron technology, to show that OCS decomposes very efficiently in DC plasmas discharges through the cleavage of the $\mathrm{C}=\mathrm{S}$ double bond, resulting in the formation of $\mathrm{CO}+\mathrm{S}$, while more complex reaction pathways resulted in small concentrations of CS and SO, and the excess sulphur was deposited on the reactor surfaces. ICP discharges of OCS were studied by Bezuk et al. (1983) using mass spectrometry and optical emission spectroscopy. CO was found to be the major product, but $S$ and $S_{2}$ were detected too. According to these authors, CS is formed in its ${ }^{1} \Pi$ excited state through electron collisions with OCS and $\mathrm{OCS}^{+}$.

Nicholas \& Amodio (1982) studied OCS and $\mathrm{CS}_{2}$ decomposition in a RF discharge. Their results corroborated the primary dissociation steps of OCS reported by previous authors, and showed that the main dissociation pathway for $\mathrm{CS}_{2}$ should be $\mathrm{e}^{-}+\mathrm{CS}_{2} \rightarrow \mathrm{CS}+\mathrm{S}+\mathrm{e}^{-}$. More recent studies on $\mathrm{CS}_{2}$ plasmas have been devoted to their application as sources of functional polymers (Ochiai \& Endo 2005, and references therein) or as a method for $\mathrm{CS}_{2}$ waste removal (Holub et al. 2014; Yan et al. 2013; Tsai et al. 2007).

We produced cold plasmas of pure OCS and pure $\mathrm{CS}_{2}$ at 0.15 mbar. In the case of OCS, different RF powers (20-100 W) in gas flow conditions produced steady state concentrations of the precursor between $40 \%$ and $14 \%$ of its initial value. The steady state concentration of $\mathrm{CS}_{2}$ with a $50 \mathrm{~W}$ discharge was $40 \%$. Mass spectra showed the formation of $\mathrm{CO}$ in OCS plasmas, as the major stable by-product. $\mathrm{S}$ was also observed as a minor product. In both plasmas, the CS radical was detected through its rotational emission in the gas phase with a $S / N>10$ in a few seconds of integration time (see Fig. 14). The observation of $\mathrm{CO}$ and $\mathrm{CS}$ in these plasmas is consistent with previous studies discussed above. For static gas plasma, the precursor and the products were quickly destroyed, a fact confirmed by the pressure decrease in the chamber and by the mass spectrum. We could, however, observe CS during several 
seconds before the precursor was completely destroyed. In this case the pressure decrease is related to deposition of the gas phase particles on the walls. In fact, a very thin layer of material was observed after a few minutes on the walls and the windows. Moreover, dust particles were observed easily floating in the plasma through the scattering of a laser beam inside the chamber. In flowing gas conditions the walls were also covered by a thin layer of sulphur compounds but at lower rate than in the static case. In this case the CS line was observed maintaining its intensity during the experiment.

Figure 14 shows the CS line observed in a flowing OCS plasma for a long integration of $120 \mathrm{~s}$. Similar results were obtained with the plasma of $\mathrm{CS}_{2}$. Figure 12 shows the $J=4-3$ line of OCS with the cell filled with OCS at 0.15 mbar before (red spectrum) and after (blue spectrum) plasma ignition. The line intensity decreases as well as the linewidth because of the drop in the total pressure of the chamber. Moreover, the two lines of the $v_{2}$ vibrational mode decrease less than the line from the ground state, from which we derive that the kinetic temperature in the chamber increases from 300 to $\sim 450 \mathrm{~K}$. This demonstrates that the HEMT receivers are sensitive enough to trace low abundance species and to monitor the kinetic temperature of the gas through the observation of the vibrationally excited modes of the molecules in the cell. For example, with a total observing time of $10 \mathrm{~min}$, lines as weak as $0.1 \mathrm{~K}$ could be detected with a signal to noise ratio of seven as it is the case of $\mathrm{OC}^{34} \mathrm{~S}$ shown in Fig. 12. The observation corresponds to a partial pressure of $\mathrm{OC}^{34} \mathrm{~S}$ of $0.025 \mathrm{mbar}$ and a total pressure of $0.5 \mathrm{mbar}$. The detection limit will depend on the dipole moment of the molecule, the partial and total pressures, and the line strength of the observed transition.

It is worth mentioning the great difficulty to monitor the CS reactive species by differentially pumped mass spectrometry, due to its loss of concentration by recombination in the path to the mass spectrometer and since most of the detected signal at mass 44 belongs to the fragment produced by electron impact on $\mathrm{CS}_{2}$ or OCS in the ionization chamber of the mass spectrometer. Thus the information provided by the emission spectrum is highly valuable.

We also note that, when only one emission line is observed for a given species, there might some uncertainty in the partial pressure derived from the emission measurements, due to the possible different rotational and vibrational temperatures. Although rotational temperatures are most likely equilibrated at the kinetic temperature of the gas and the total pressures employed in these experiments, the vibrational temperatures might depart of the kinetic temperature upon the RF excitation.

We used an $\mathrm{O}_{2}$ plasma $(0.1 \mathrm{mbar}, 50 \mathrm{~W})$ to clean the walls of the cavity which were covered, after the OCS and $\mathrm{CS}_{2}$ plasma, by a thin layer deposit of a browny material mainly containing sulphur. During the cleaning process we searched for possible species produced in the cell walls, $\mathrm{SO}$ and $\mathrm{SO}_{2}$. SO has no transitions within the $Q$ band and therefore only $\mathrm{SO}_{2}$ was searched for. Figure 15 shows one of the lines of $\mathrm{SO}_{2}$ observed during the $\mathrm{O}_{2}$ plasma. Before the $\mathrm{O}_{2}$ plasma was initiated, the chamber was pumped down to $10^{-5}$ mbar. Hence, the only sulphur available to produce $\mathrm{SO}_{2}$ was on the walls. The partial pressure of $\mathrm{SO}_{2}$ from the observed integrated line intensity was derived to be $\sim 0.04$ mbar. The mass spectra of the $\mathrm{O}_{2}$ discharge also showed the formation of $\mathrm{SO}_{2}$, whereas the $\mathrm{CO}$ signal arising from possible carbon deposition during the previous OCS and $\mathrm{CS}_{2}$ plasmas was practically negligible.

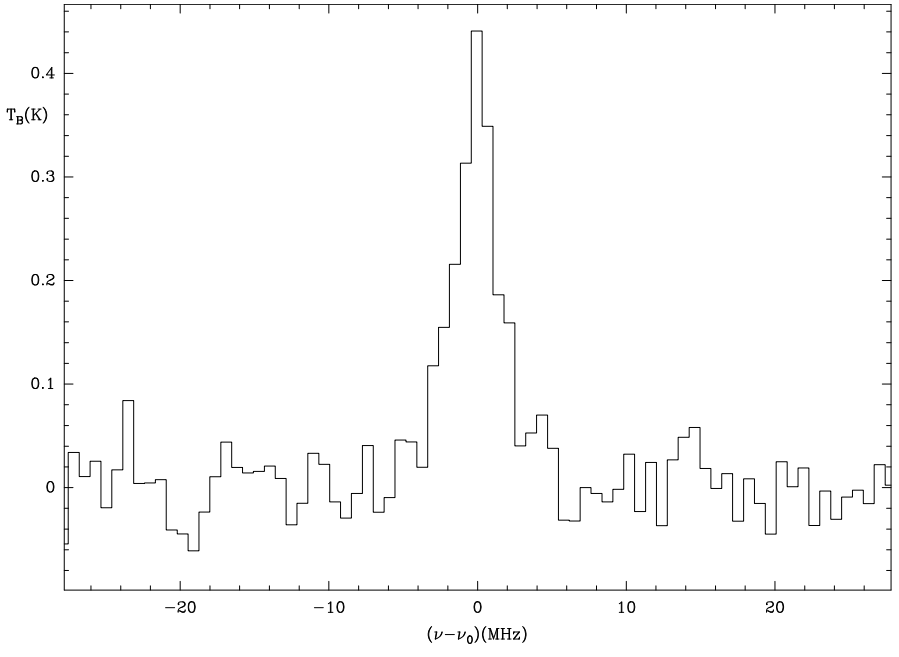

Fig. 15. Observed $\mathrm{SO}_{2} 14_{2,12}-13_{3,11}$ signal (centred at $47913.427 \mathrm{MHz}$ ) during the cleaning of the cell through an $\mathrm{O}_{2}$ plasma at 0.1 mbar. The integration time was $5 \mathrm{~min}$. The derived partial pressure of $\mathrm{SO}_{2}$ is 0.04 mbar (see text). The spectral resolution is $640 \mathrm{kHz}$.

\subsection{Photodissociation and photochemistry}

The first experiment with the UV lamp was performed with $\mathrm{CS}_{2}$. Figures 2, 3 show the experimental configuration. The gas cell was filled with $\mathrm{CS}_{2}$ at a pressure of 0.4 mbar. The illumination with the UV lamp was maintained during the total duration of the experiment with data acquired every $20 \mathrm{~s}$ with a blanking time of five seconds imposed by the software of the telescope. A reference spectrum was performed at the beginning of the experiment by observing the empty cell during 200 s. Hence, the noise in the data arises essentially from the ON phase of each spectra. The total observing time was $2260 \mathrm{~s}$ corresponding to 77 spectra (see Fig. 16 and associated video). Several processes are expected to occur during the experiment. First, as discussed below, $\mathrm{CS}_{2}$ could dissociate into $\mathrm{CS}$ and $\mathrm{S}$. Both species can react with $\mathrm{CS}_{2}$ and with the walls of the cell. In turn, CS could be photodissociated into $\mathrm{C}$ and $\mathrm{S}$.

Although wall effects have to be considered in order to study the whole chemistry in the cell, we have noticed that without UV light the $\mathrm{CS}_{2}$ remains in gas phase as measured with the mass spectrometer for times much longer (the longest experiment under these conditions was one hour) than those of the experiment with UV light (minutes). The data shown in Fig. 16 indicate that CS reaches its maximum abundance at $\sim 200 \mathrm{~s}$, decreasing smoothly afterwards. The observed maximum intensity corresponds to a partial pressure of CS, $P(\mathrm{CS})$, of $\sim 10^{-3}$ mbar. The line broadening is produced essentially by the remaining gas, which, according to mass spectrometric data, is composed mainly of $\mathrm{CS}_{2}$, which partially photodissociates, and water desorbed from the walls under UV irradiation, maintaining nearly a constant total pressure, during the experiment.

The gas-phase UV absorption spectroscopy of $\mathrm{CS}_{2}$ has been extensively studied since 1969 in a wide wavelength range (31-500 nm; Keller-Rudek et al. 2013, and references therein). Recent high-resolution absorption measurements in the 105-225 nm region (Sunanda et al. 2015) and in the 205-370 nm (Grosch et al. 2015) reveal that the spectrum of $\mathrm{CS}_{2}$ presents a highly structured band in the VUV region, with a maximum absorption cross section of $3.84 \times$ $10^{-16} \mathrm{~cm}^{2}$ molecule ${ }^{-1}$ located at $198.26 \mathrm{~nm}$ at room temperature (Sunanda et al. 2015). For that reason, photodissociation of $\mathrm{CS}_{2}$ 

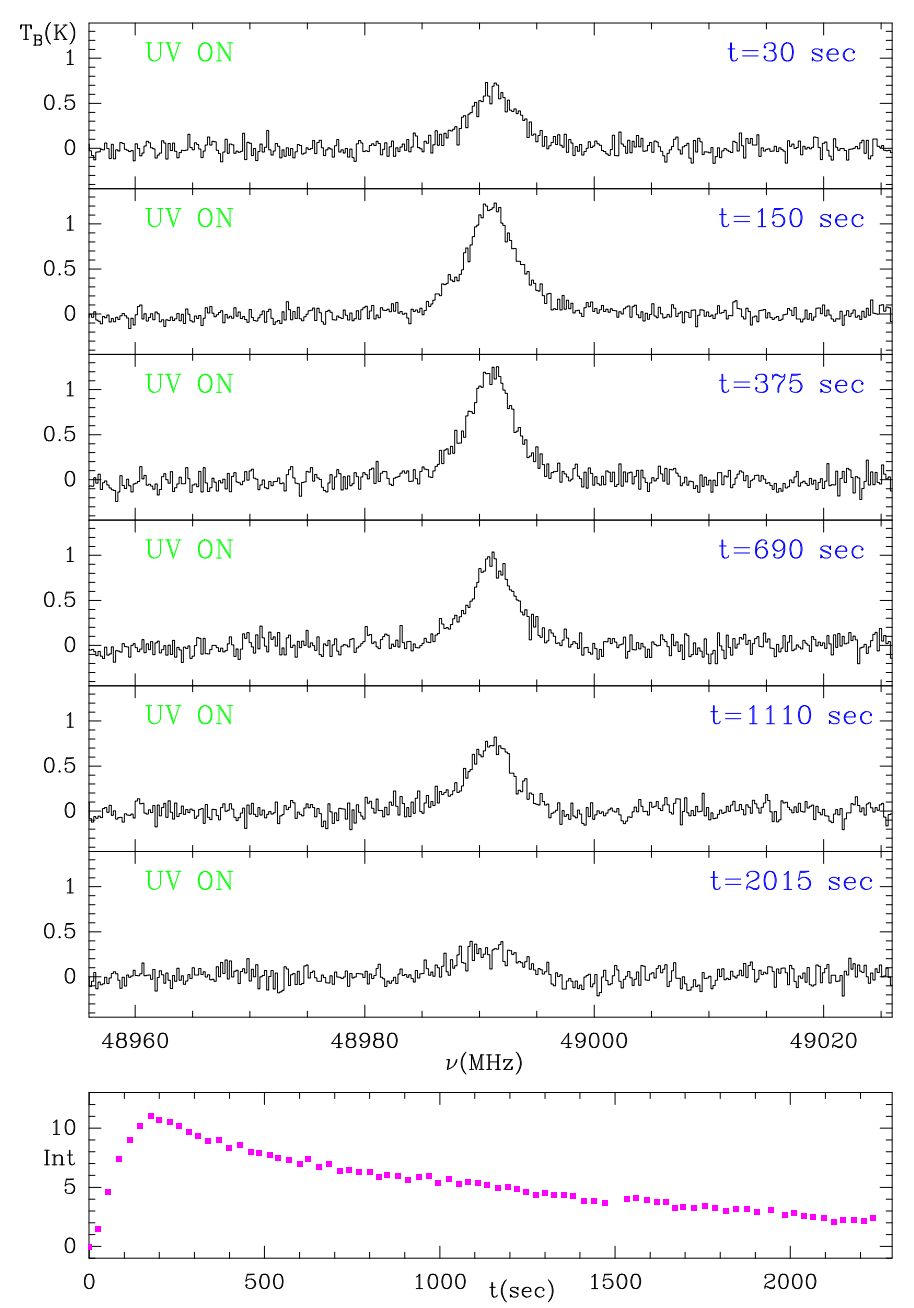

Fig. 16. Observed $J=1-0 \mathrm{CS}$ transition at $48990.957 \mathrm{MHz}$ using the UV lamp. The integration time was $20 \mathrm{~s}$ per spectra. The spectral resolution of the data is $190 \mathrm{kHz}$. The upper panels show spectra at selected times while the lower panel shows the line integrated intensity $(\mathrm{K} \times \mathrm{MHz})$ as a function of time. A video showing the whole set of data is available online.

is efficient at the $193 \mathrm{~nm}$ excimer laser wavelength which produces $\mathrm{CS}\left(\mathrm{X}^{1} \Sigma^{+}, v=1\right.$ to $5, J=5$ to 45$), \mathrm{S}\left({ }^{3} \mathrm{P}\right)$ and $\mathrm{S}\left({ }^{1} \mathrm{D}\right)$ fragments (Yang et al. 1980; Butler et al. 1980). Kitsoupolos et al. (2001) determined the $S\left({ }^{3} \mathrm{P}_{2,1,0}\right) / \mathrm{S}\left({ }^{1} \mathrm{D}_{2}\right)$ branching ratio to be $1.5 \pm 0.4$ from the analysis of the speed distributions of the photofragments. In our photochemical studies the UV lamp provides emission lines at $185 \mathrm{~nm}$ and $254 \mathrm{~nm}$ at which absorption cross sections of $\mathrm{CS}_{2}$ are $2.32 \times 10^{-17} \mathrm{~cm}^{2}$ molecule ${ }^{-1}$ (Sunanda et al. 2015) and $<10^{-22} \mathrm{~cm}^{2}$ molecule ${ }^{-1}$ (Grosch et al. 2015), respectively. Hence, in our experiments $\mathrm{CS}_{2}$ is expected to be almost exclusively photolyzed by the $185 \mathrm{~nm}$ radiation.

In order to provide some control on the wall effects we repeated the experiment but the UV lamp was switched off after $\mathrm{CS}$ reached its maximum (at $200 \mathrm{~s}$ ). Figure 17 shows the resulting data. At $100 \mathrm{~s}$ after switching off the lamp, the partial pressure of CS measured from the intensity of the $J=1-0$ line decreased by a factor of two. After $200 \mathrm{~s}$, we could only derive upper limits to $P(\mathrm{CS})$. Hence, a long survival time of $\mathrm{CS}$ in the gas phase is observed after its production by photodissociation is interrupted.

In a second experiment, we checked the feasibility of chemical reactions in the cell induced by photochemistry by
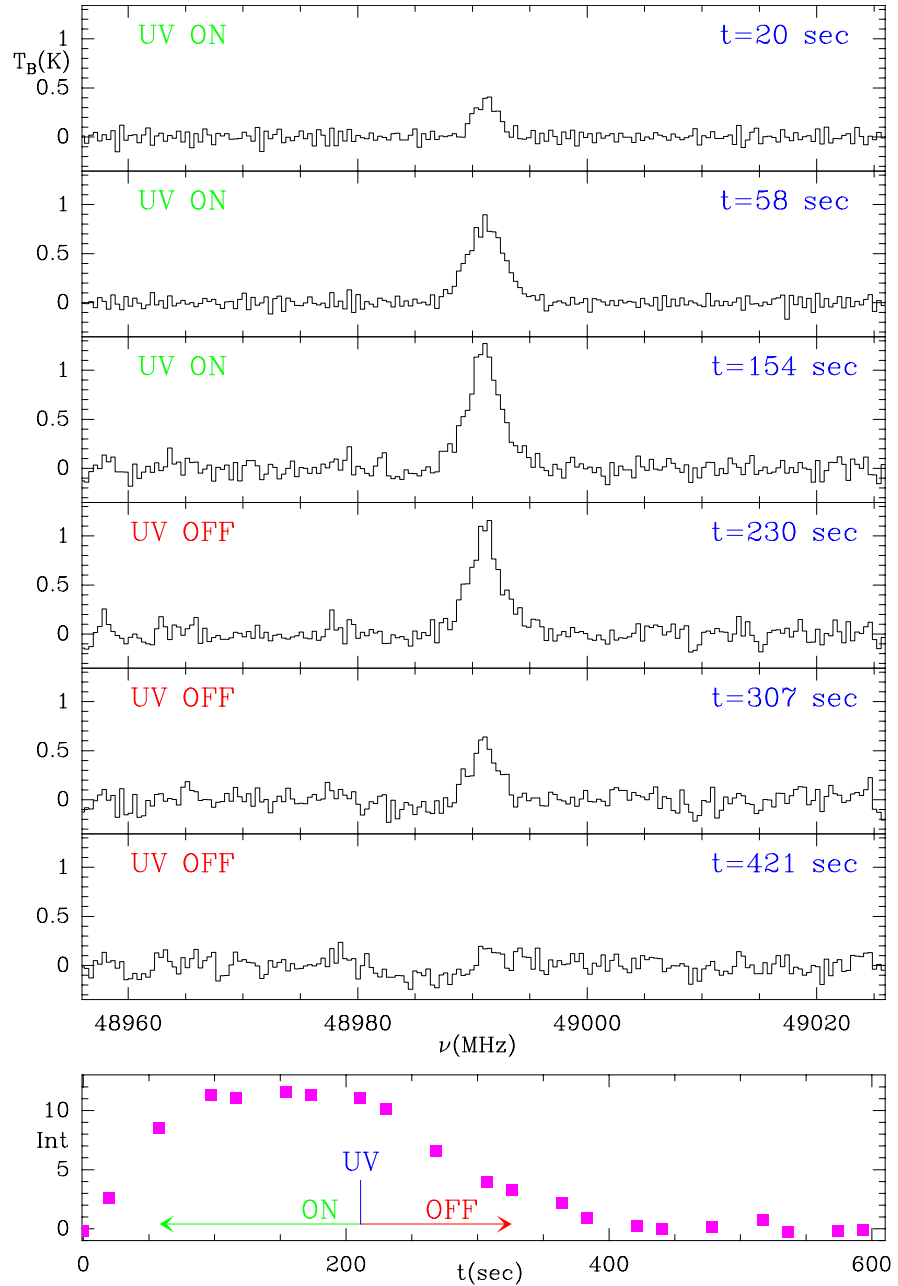

Fig. 17. Observed $J=1-0 \mathrm{CS}$ transition at $48990.957 \mathrm{MHz}$ using the UV lamp. The integration time was $20 \mathrm{~s}$ per spectra. The spectral resolution of the data is $380 \mathrm{kHz}$. The upper panels show spectra at selected times while the lower panel shows the line integrated intensity (in $\mathrm{K} \times \mathrm{MHz}$ ) as a function of time. The lamp was switched off after $200 \mathrm{~s}$. The decrease of the CS abundance is much faster than in the case of continuous illumination of the cell. A video showing the whole set of data is available online.

introducing 0.4 mbar of $\mathrm{CS}_{2}$ and 0.2 mbar of $\mathrm{O}_{2}$ in the presence of UV radiation. Considering the corresponding absorption cross sections at $185 \mathrm{~nm}\left(2.038 \times 10^{-21} \mathrm{~cm}^{2}\right.$ molecule ${ }^{-1}$ for $\mathrm{O}_{2}$, Yoshino et al. 1992; and $2.32 \times 10^{-17} \mathrm{~cm}^{2}$ molecule ${ }^{-1}$ for $\mathrm{CS}_{2}$, Sunanda et al. 2015), CS radicals are expected to be formed, as well as $\mathrm{O}$ atoms but at a lower extend. The expected molecules to be detected, in addition of CS, are OCS, $\mathrm{SO}, \mathrm{O}_{3}$, and $\mathrm{SO}_{2}$. Unfortunately, as mentioned before, $\mathrm{SO}$ does not have rotational transitions in the spectral range of the receiver of the Aries- $40 \mathrm{~m}$. The $J=1-0$ line of CS and the $J=4-3$ line of OCS are separated by $240 \mathrm{MHz}$ and were observed simultaneously using the same procedure than in previous cases. Figure 18 shows the results. After CS reaches its maximum abundance, OCS starts to appear. The partial pressure of CS is again $\sim 0.001$ mbar while that of OCS is $\sim 0.002$ mbar at times larger than $150 \mathrm{~s}$.

The $\mathrm{O}_{2}+\mathrm{CS}_{2}$ reaction has been measured in the laboratory at temperatures between 1500 and $2100 \mathrm{~K}$ and was found to exhibit a very high barrier, $\sim 16000 \mathrm{~K}$ (Saito et al. 1986). Hence, its contribution to the chemistry in the cell at $300 \mathrm{~K}$ is expected to be negligible. The reaction of $\mathrm{O}_{2}$ with $\mathrm{CS}$ has two product 


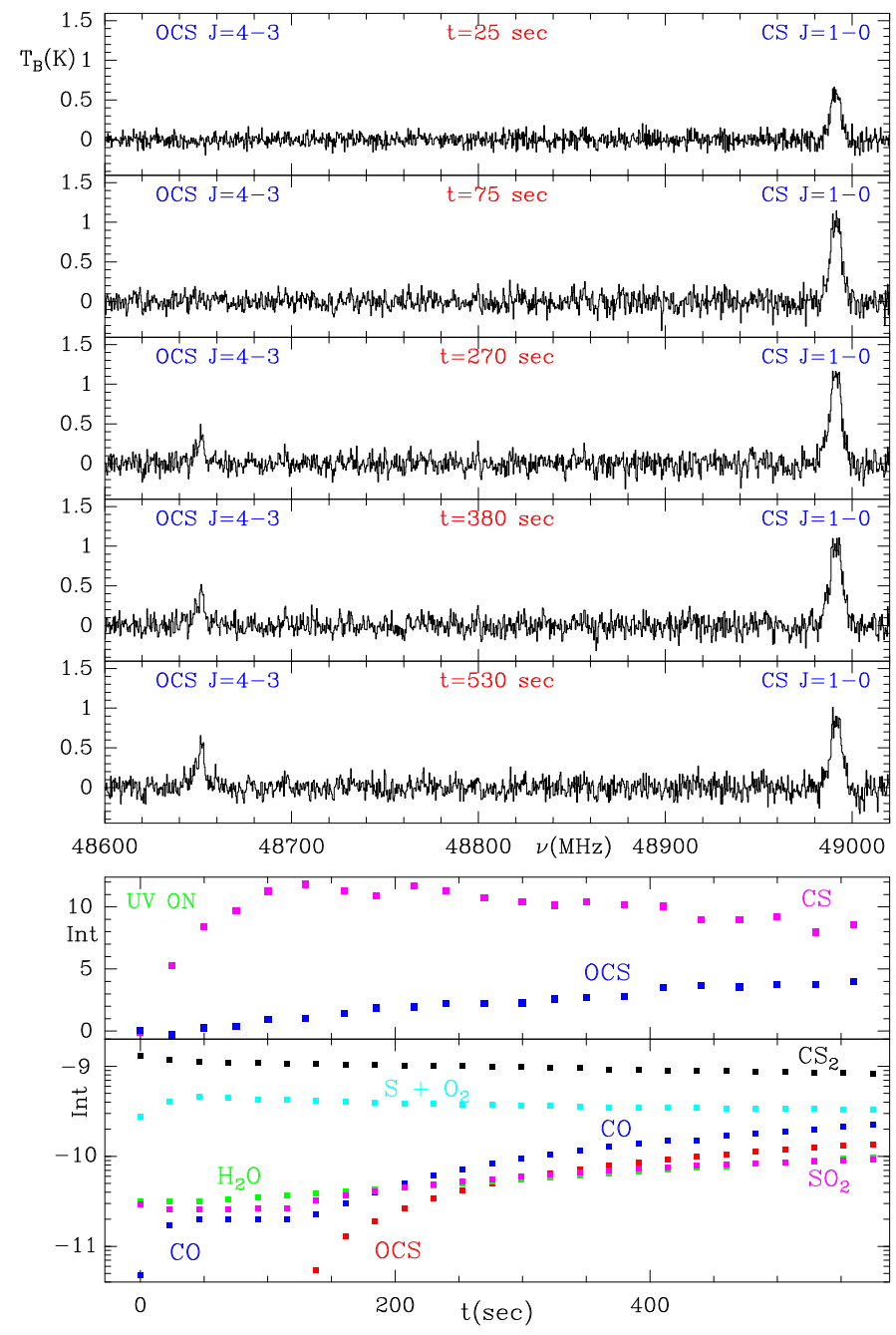

Fig. 18. Observed transitions CS $J=1-0$ and OCS $J=4-3$ transitions using the UV lamp. The integration time was $20 \mathrm{~s}$ per spectrum. The spectral resolution of the data is $380 \mathrm{kHz}$. The upper panels show spectra at selected times while the intermediate panel shows the line integrated intensity (in $\mathrm{K} \times \mathrm{MHz}$ ) of CS and OCS as a function of time. Finally, the bottom panel shows the intensity derived from the mass spectrum for different masses $\left(\mathrm{CS}_{2}, \mathrm{OCS}, \mathrm{H}_{2} \mathrm{O}, \mathrm{S}\right.$ and $\mathrm{O}_{2}, \mathrm{SO}_{2}$, and $\mathrm{CO}$ ). A video showing the whole set of data is available online.

channels (Breckenridge et al. 1975), one producing OCS + O with a very low rate coefficient $(k)$ at ambient temperature of $k=2.9 \times 10^{-19} \mathrm{~cm}^{3} \mathrm{~s}^{-1}$ (Atkinson et al. 1997), the other producing $\mathrm{CO}+\mathrm{SO}$ with $k=3.01 \times 10^{-18} \mathrm{~cm}^{3} \mathrm{~s}^{-1}$ at room temperature. Therefore, formation of OCS from the reaction of CS with molecular oxygen is negligible in the gas phase. Alternative mechanisms for the production of OCS include wall effects and reaction of atomic oxygen, product of the photodissociation of $\mathrm{O}_{2}$, with $\mathrm{CS}_{2}$.

Atomic oxygen reacts with $\mathrm{CS}_{2}$ at $300 \mathrm{~K}$ to produce

- CS + SO, $k=3.75 \times 10^{-12} \mathrm{~cm}^{3} \mathrm{~s}^{-1}$ (Singleton \& Cvetanovic 1988).

- OCS $+\mathrm{S}, k=3.06 \times 10^{-13} \mathrm{~cm}^{3} \mathrm{~s}^{-1}$ (Cooper \& Hershberger 1992).

- $\mathrm{CO}+\mathrm{S}_{2}, k=1.08 \times 10^{-13} \mathrm{~cm}^{3} \mathrm{~s}^{-1}$ (Cooper \& Hershberger 1992).
Contrarily to molecular oxygen, atomic oxygen also reacts relatively fast at ambient temperature with CS:

$\mathrm{O}+\mathrm{CS} \rightarrow \mathrm{CO}+\mathrm{S}$

with $k=2.11 \times 10^{-11} \mathrm{~cm}^{3} \mathrm{~s}^{-1}$ (Atkinson et al. 1997). Hence, if atomic oxygen is present with high abundance in the gas phase it will produce OCS, but also distroy most CS obtained from the photodissociation of $\mathrm{CS}_{2}$. On the other hand, the reaction of atomic oxygen with OCS proceeds very slowly, $k=$ $1.36 \times 10^{-14} \mathrm{~cm}^{3} \mathrm{~s}^{-1}$ (Singleton \& Cvetanovic 1988). Hence OCS, if formed from the reaction of $\mathrm{O}$ and $\mathrm{CS}_{2}$, could survive in the gas phase, being only destroyed by wall reactions and by UV photons.

The mass spectra obtained during the experiment help to understand the chemical processes occurring in the cell. The main observed species are $\mathrm{CS}_{2}$ (76), $\mathrm{SO}_{2}$ (64), OCS (60), $\mathrm{S} / \mathrm{O}_{2}$ (32), $\mathrm{CO}$ (28), $\mathrm{H}_{2} \mathrm{O}$ (18). At the beginning, a fast increase of mass peak 32 is observed due to the photodissociation of $\mathrm{CS}_{2}$ which overcompensates the photodissociation of $\mathrm{O}_{2}$. Then, both $\mathrm{CS}_{2}$ and mass peak 32 decrease by a factor of two during the experiment. We note that OCS, as traced by the emission of its $J=4-3$ line, requires nearly $150 \mathrm{~s}$ to reach an abundance high enough to be detected with the receivers. A similar time is also required to produce a significant signal in the mass spectrometer for OCS. The first sharp increase of $\mathrm{CO}$ is associated to reaction (20). Later, once OCS is formed then CO can be produced by the photodissociation of OCS. The threshold for photodissociation of OCS is near $255 \mathrm{~nm}$ (Lochte-Holtgreven \& Bawn 1932) and the photofragments produced are CO, in its electronic ground state, and sulphur atoms in its ${ }^{1} \mathrm{D}_{2}$ state. In our experiment the photodissociation is dominated by the absorption of the $185 \mathrm{~nm}$ line which has a cross section of $\sim 3 \times$ $10^{-19} \mathrm{~cm}^{2}$ molecule ${ }^{-1}$ (Keller-Rudek et al. 2013).

The mass peak corresponding to $\mathrm{H}_{2} \mathrm{O}$ (which is certainly desorbed from the walls) shows a flat distribution following a very similar time dependence to that of $\mathrm{SO}_{2}$ which is probably formed on the walls, since the gas phase reaction

$\mathrm{SO}+\mathrm{O}_{2} \rightarrow \mathrm{SO}_{2}+\mathrm{O}$

proceeds very slowly at ambient temperature, $k \sim 8 \times$ $10^{-17} \mathrm{~cm}^{3} \mathrm{~s}^{-1}$ (Atkinson et al. 1997; DeMore et al. 1997).

$\mathrm{O}_{3}$ was not observed in our experiment. However, in the Schumann-Runge band spectral region (175-200 nm), $\mathrm{O}_{2}$ can be photolyzed forming $\mathrm{O}$ atoms via these two dissociative channels:

$\mathrm{O}_{2}\left(\mathrm{X}^{3} \Sigma^{-}\right)+h v(\lambda<240 \mathrm{~nm}) \rightarrow \mathrm{O}\left({ }^{3} \mathrm{P}\right)+\mathrm{O}\left({ }^{1} \mathrm{D}\right)$,

and $\mathrm{O}\left({ }^{3} \mathrm{P}\right)+\mathrm{O}\left({ }^{3} \mathrm{P}\right)$. At $\lambda<177 \mathrm{~nm}$, the quantum yield of $\mathrm{O}\left({ }^{1} \mathrm{D}\right)$ is unity, while it is zero at longer wavelengths (Nee \& Lee 1997). Therefore, oxygen atoms are formed in the ground state, ${ }^{3} \mathrm{P}$, when $\mathrm{O}_{2}$ is photodissociated at $\lambda=185 \mathrm{~nm}$.

$\mathrm{O}\left({ }^{3} \mathrm{P}\right)$ atoms can react rapidly with $\mathrm{O}_{2}$ in a three-body reaction to form ozone, $\mathrm{O}_{3}$ :

$\mathrm{O}\left({ }^{3} \mathrm{P}\right)+\mathrm{O}_{2}+\mathrm{M} \rightarrow \mathrm{O}_{3}+\mathrm{M}$,

where $\mathrm{M}$ is a third body $\left(\mathrm{O}_{2}\right.$ or $\left.\mathrm{CS}_{2}\right) . \mathrm{O}_{3}$ is photodissociated efficiently by the radiation at $254 \mathrm{~nm}$ of the mercury lamp:

$\mathrm{O}_{3}+\mathrm{h} v(254 \mathrm{~nm}) \rightarrow \mathrm{O}\left({ }^{1} \mathrm{D}\right)+\mathrm{O}_{2}$,

followed by

$\mathrm{O}\left({ }^{1} \mathrm{D}\right)+\mathrm{M} \rightarrow \mathrm{O}\left({ }^{3} \mathrm{P}\right)+\mathrm{M}$. 
We can therefore understand why $\mathrm{O}_{3}$ was not observed in our cell. First, the three body reaction producing it proceeds slowly at our low experimental pressures. In addition, the lamp flux at $254 \mathrm{~nm}$ is about five times that at $185 \mathrm{~nm}$, and the absorption cross section of $\mathrm{O}_{3}$ at $254 \mathrm{~nm}$ is $1.157 \times 10^{-17} \mathrm{~cm}^{2}$ molec $^{-1}$ (Molina \& Molina 1986). Consequently, ozone concentration in the mixture is expected to be very small and, furthemore, the intensity of the lines is rather low $(I(300 \mathrm{~K})=9.72 \times$ $10^{-7} \mathrm{~nm}^{2} \mathrm{MHz}$ for the $1_{1,1}-2_{0,2}$, the strongest line in this region). Hence, the expected intensities for these ozone lines are below our detection limit in the present experiments.

A detailed analysis of the different involved chemical processes would require additional experiments at different partial pressures of $\mathrm{CS}_{2}$ and $\mathrm{O}_{2}$, and different intensities of the UV lamp. Nevertheless, the three experiments shown in this section demonstrates the capacity of radio astronomical receivers to perform chemical experiments of astrophysical interest.

\section{Conclusions}

In this work we have shown that radio astronomical observation techniques and receivers can be used in laboratory experiments to perform high-sensitivity molecular spectroscopy of molecules inside a gas cell at low pressure. Compared with other techniques at low frequency the sensitivity offered by radio astronomical receivers is not as performant and will require longer integration times. However, even at these frequencies, the very large instantaneous bandwidth (around $20 \mathrm{GHz}$ ), spectral purity, and the linear dependence of line intensity with partial pressures, provides an alternate way to perform experiments of interest in the field of astrochemistry and laboratory spectroscopy. At millimeter and submillimeter wavelengths, with an instantaneous bandwidth of $40 \mathrm{GHz}$ and a high spectral purity, emission spectroscopy becomes comparable in sensitivity with narrow band laboratory absorption techniques and permits to follow the time variation of many species simultaneously in chambers devoted to laboratory simulations of the chemistry of interstellar clouds.

The selection of appropriated windows for the cell has been addressed. Standard quartz windows, which would be a natural choice in ultra-high vacuum conditions, are not adapted to reach the sensitivity provided by the heterodyne receivers. The interference between the two faces of the quartz window worsens performance and appropriately designed anti-reflection coatings are important. If total pressures above $10^{-4}$ mbar prevail in the cell, then Upilex windows can be used providing a very good transmission and a minimum degradation of the receiver performance. Nevertheless, other materials with appropriate antireflection treatments will be tested. First results using Teflon windows indicate that this material offers very good transmission and safety conditions.

We showed that molecular spectroscopy can be performed in the laboratory by using radio astronomical receivers and spectrometers, which are coupled with a small size gas cell placed filled with a static (stable molecules) or flowing gas (for unstable species). Standard ON/OFF or FSW observing modes can be performed depending on the type and goals of the experiments. Special care has to be taken with the stability of the cold load.

Radio frequency cold plasmas can be produced in the gas cell without any perturbation of the receivers and their associated electronics. We have generated cold plasmas of different molecular species and successfully detected partial pressures of their products as low as $10^{-3}$ mbar.

UV photochemistry can be studied in the cell. Partial pressures of photochemical products as low as $10^{-3}$ mbar have been observed in our experiments. No perturbation has been found between the lamp discharge and the receiver electronics.

The residence time in the gas phase of reactive species produced in plasma or the photolysis experiments, such as CS, is large enough to allow further chemical reactions with potential in studying the chemistry of photodissociation regions in the ISM. To conclude, we expect that the gas cell that will be soon operational for the Nanocosmos project, $1 \mathrm{~m}$ long, with the $31.5-50$ and $72-116.5 \mathrm{GHz}$ frequency domain covered by HEMT receivers, coupled to $40 \mathrm{GHz}$ wide Fast Fourier Transform Spectrometers, will open new perspectives in laboratory astrophysics.

Acknowledgements. The research leading to these results has received funding from the European Research Council under the European Union's Seventh Framework Programme (FP/2007-2013)/ERC-SyG-2013 Grant Agreement No. 610256 NANOCOSMOS and from spanish MINECO CSD2009-00038 (ASTROMOL) under the Consolider-Ingenio Program. We also thank spanish MINECO for funding under grants AYA2012-32032, AYA2016-75066-C2-1-P, FIS2013-48087-C2-1-P, FIS2016-77726-C3-1-P, FIS2016-77578-R, MAT201454231-C4-1-P.

\section{References}

Abeysekera, C., Zack, L. N., Barratt, P. G., et al. 2014, J. Chem. Phys., 141, 214203

Atkinson, R., Baulch, D. L., Cox, R. A., et al. 1997, J. Phys. Chem. Ref. Data, 26, 1329

Bezuk, S. J., Miller, L. L., \& Platzer, I. 1983, J. Phys. Chem., 87, 131

Breckenridge, W. H., Kolln, W. S., \& Moore, D. S. 1975, Chem. Phys. Lett., 32, 290

Brown, G. G., Seely, J. F., Feldman, U., et al. 2008, Rev. Sci. Instrum, 79, 053103 Butler, J. E., Drozdoski, W. S., \& McDonald, J. R. 1980, Chem. Phys., 50, 413

Cernicharo, J. 1985, ATM a code to compute atmospheric opacity up to $1 \mathrm{THz}$, IRAM internal report

Cernicharo, J. 2012, in ECLA-2011: Proc. of the European Conference on Laboratory Astrophysics, EAS Publ. Ser., 2012, eds. C. Stehl, C. Joblin, \& L. d'Hendecourt (Cambridge: Cambridge Univ. Press), 251

Cooper, W. F., \& Hershberger, J. F. 1992, J. Phys. Chem., 96, 5405

Clark, W. W., \& De Lucia, F. C. 1981, J. Chem. Phys., 74, 3139

DeMore, W. B., Sander, S. P., Golden, A., et al. 1997, JPL Publication, 07-4

Dian, B. C., Brown, G. G., Douglass, K. O., \& Pate, B. H. 2008, Science, 320, 924

Frisk, U., Hagström, M., Ala- Laurinaho, J., et al. 2003, A\&A, 402, L27

Gerecht, E., Douglass, K. O., \& Plusquellic, D. F. 2011, Opt. Exp., 19, 8973

Grosch, H., Fateev, A., \& Clausen, S. 2015, J. Quant. Spectr. Rad. Transf., 154, 28

Hartmann, J.-M., Boulet, C., \& Robert, D. 2008, Collisional Effects on Molecular Spectra (Amsterdam: Elsevier)

Higgins, R. D. 2011, Ph.D. Lecture, University of Nui Maynooth

Higgins, R. D., Teyssier, D., Pearson, J. C., et al. 2010, in Twenty-First International Symposium on Space Terahertz Technology, National Radio Astronomy Observatory (NRAO), 390

Holub, M., Brandenburg, R., Grosch, H., Weinmann, S., \& Hansel, B. 2014, Aerosol and Air Quality Research, 14, 697

Hüberts, H. W., Keilmann, F., Wittborn, J., et al. 2006, Appl. Phys. Lett., 89, 061115

Keller-Rudek, H., Moortgat, G. K., Sander, R., \& Sörensen, R. 2013, Earth Syst. Sci. Data, 5, 365

Kitsopoulos, T. N., Gebhardt, C. R., \& Rakitzis, T. P. 2001, J. Chem. Phys., 115, 9727

Koshelev, M., \& Tetryakov, M. Y. 2009, J. Quant. Spectr. Rad. Transf., 110, 118 Lochte-Holtgreven, W., \& Bawn, C. E. H., 1932, Trans. Faraday Soc., 28, 698

Molina, L. T., \& Molina, M. J. 1986, J. Geophys. Res., 91, 14501

Müller, H. S. P., Schlöder, F., Stutzki, J., \& Winnewisser, G. 2005, J. Mol. Struct., 742,215

Nee, J. B., \& Lee, P. C. 1997, J. Phys. Chem. A, 101, 6653

Neumaier, P. F. X., Richter, H., Stake, J., et al. 2014, IEEE Trans. TeraHertz Sci. Tech., 4, 469

Nicholas, J. E., \& Amodio, C. A. 1982, Plasma Chem. Plasma Process., 2, 331

Ochiai, B., \& Endo, T. 2005, Progress in Polymer Science, 30, 183

Pardo, J. R., Cernicharo, J., \& Serabyn, E. 2001, IEEE Trans. Antennas and Propagation, 49/12, 1683 
I. Tanarro et al.: A gas cell for astrochemical simulations

Park, G. B., Steeves, A. H., Kuyanov-Prozument, K., et al. 2011, J. Chem. Phys., 135, 024202

Pickett, H. M., Poynter, R. L., Cohen, E. A., et al. 1998, J. Quant. Spectr. Rad. Transf., 60, 883

Ren, Y., Klaassen, T. O., Williams, B. S., et al. 2010, Appl. Phys. Lett., 97, 161105

Saito, K. I., Ueda, Y., Kakumoto, T., \& Imamura, A. 1986, Int. J. Chem. Kinet., 18,871

Schmitz, D., Shubert, V. A., Betz, T., \& Schnell, M. 2012, J. Mol. Spectr., 280,

Singleton, D., \& Cvetanovic, R. J. 1988, J. Phys. Chem. Ref. Data, 17, 1377 Sunanda, K., Shastri, A., Das, A. K., \& Raja Sekhar, B. N. 2015, J. Quant. Spectr. Rad. Transf., 151, 76
Tsai, C. H., Lin, Y. C., Chen, H. Y., \& Tsai, P. S. 2007, Env. Engin. Sci., 25, 821 Teyssier, D., Dartois, E., Crussaire, J. P., et al. 2004, Proc. Fifteenth International Symposium on Space Terahertz Technology, held April 27-29, 2004, in Northampton, Massachusetts, USA, ed. G. Narayanan, 306

Tolls, V., Melnick,, G. J., Ashby, M. L. N., et al. 2004, ApJS, 152, 137

Wehres, N., Maßen, J., Borisov, K. et al. 2017, PCCP, in press DOI: $10.1039 / \mathrm{C} 7 \mathrm{CP} 06394 \mathrm{~F}$

Yan, X., Sun, Y., Zhu, T., \& Fan, X. 2013, J. Hazardous Materials, 261, 669

Yang, S. C., Freedman, A., Kawasaki, M., \& Bersohn, R. 1980, J. Chem. Phys., 72,4058

Yoshino, K., Esmond, J. R., Cheung, A. S.-C., Freeman, D. E., \& Parkinson, W. H. 1992, Planet. Space Sci., 40, 185 


\section{Appendix A: Line profiles}

The effect of the lineshape on the observed spectrum deserves some consideration because, for a given integrated line intensity, it will determine the peak value of the detected signal, and therefore the attainable signal to noise ratio for a given integration time.

The main line broadening mechanisms at the frequencies covered by the receiver and at the typical pressure and temperature operating conditions are thermal (Doppler) broadening and collisional (pressure) broadening. Neglecting more subtle effects such as Dicke narrowing, the speed dependence of the relaxation rates or line-mixing effects (Hartmann et al. 2008), the lineshape functions describing the Doppler and the collisional broadening are the Gaussian function and the Van Vleck-Weisskopf function, respectively. The normalized Doppler lineshape function can be expressed as

$\phi_{\mathrm{D}}(v)=\frac{1}{\Delta v_{\mathrm{D}}} \sqrt{\frac{\ln (2)}{\pi}} \mathrm{e}^{-\ln (2)\left(\left(v-v_{0}\right) / \Delta v_{\mathrm{D}}\right)^{2}}$,

with

$\Delta v_{\mathrm{D}}=3.581 \times 10^{-7} v_{0} \sqrt{\frac{T}{M}}$,

where $\Delta v_{\mathrm{D}}$ is the Gaussian half width at half maximum, $T$ is the temperature in $\mathrm{K}$ and $M$ the molecular mass in unified atomic mass units. As an example, at $300 \mathrm{~K}$, and at $45 \mathrm{GHz}$, the Doppler full width at half maximum (FWHM) of a molecule of $M=$ $50 \mathrm{amu}$ is $2 \Delta v_{\mathrm{D}}=79 \mathrm{kHz}$.

The normalized Van Vleck-Weisskopf lineshape profile can be written

$\phi_{\mathrm{VVW}}(v)=\frac{v}{\pi v_{0}}\left(\frac{\Delta v}{(\Delta v)^{2}+\left(v-v_{0}\right)^{2}}+\frac{\Delta v}{(\Delta v)^{2}+\left(v+v_{0}\right)^{2}}\right)$,

with $\Delta v=\left(2 \pi t_{2}\right)^{-1}$, and $t_{2}$ the average time between molecular collisions. When $v_{0} \gg \Delta v$, the Van Vleck-Weisskopf profile reduces to the Lorentz lineshape with $\Delta v=\Delta v_{\mathrm{C}}$, the HWHM of the Lorentz profile, that is

$\phi_{\mathrm{L}}(v)=\frac{1}{\pi} \frac{\Delta v_{\mathrm{C}}}{\left(v-v_{0}\right)^{2}+\Delta v_{\mathrm{C}}^{2}}$.

The collisional width in this model increases linearly with pressure (or density), i.e.: $\Delta v_{\mathrm{C}}=\Delta v_{\mathrm{C}}^{0} \times P$, where $\Delta v_{\mathrm{C}}^{0}$ is the pressure broadening coefficient normally expressed in units of $\mathrm{MHz} / \mathrm{mbar}, \mathrm{MHz} /$ Torr, or $\left.\mathrm{cm}^{-1} / \mathrm{atm}\right)$ and $P$ is the pressure. Note that pressure broadening coefficients in the literature most frequently refer to the Lorentz halfwidth at half maximum. They depend on the quantum numbers of the transition, the temperature and the colliding partner, but typical values are
2-5 MHz/mbar. In a mixture of species the collisional width is additive, that is:

$\Delta v_{\mathrm{C}}=P \sum_{\mathrm{A}} X_{A}\left(\Delta v_{\mathrm{C}}^{0}\right)_{\mathrm{A}}$,

where $P$ is the total pressure, $X_{A}$ is the mole fraction of species $A$ and $\left(\Delta v_{\mathrm{C}}^{0}\right)_{A}$ its corresponding pressure broadening coefficient. As an example, for pressures 1-0.01 mbar and a typical $\Delta v_{\mathrm{C}}^{0}=$ $3 \mathrm{MHz} / \mathrm{mbar}, \Delta v_{\mathrm{C}}=3-0.03 \mathrm{MHz}$. It can be seen that, except for rather low pressures, the dominant broadening mechanism in the cell will be collisional broadening, but with a non-negligible contribution of Doppler broadening. In this regime, and, again, neglecting speed-dependent and other effects, the Voigt profile renders a good representation of the spectral shape. Moreover, for the highest pressures where the cell will be operated (a few mbar), the Lorentz lineshape can be used.

Mathematically, the Voigt profile is the convolution of a Gaussian and a Lorentzian function, and, physically, implies that both broadening mechanisms are totally uncorrelated, i.e., that each velocity class has its own spectral distribution owing to collisions. The Voigt profile has no analytical expression, but it can be written as

$\phi_{V}(v)=\sqrt{\frac{\ln (2)}{\pi}} \frac{1}{\Delta \nu_{\mathrm{D}}} \frac{a}{\pi} \int_{-\infty}^{+\infty} \frac{\mathrm{e}^{-y^{2}}}{a^{2}+(w-y)^{2}} \mathrm{~d} y$,

with $w=\sqrt{\ln (2)}\left(v-v_{0}\right) / \Delta v_{\mathrm{D}}$ and $a=\sqrt{\ln (2)} \Delta v_{\mathrm{C}} / \Delta v_{\mathrm{D}}$. Combining $a$ and $w$ into the complex variable $z=a+\mathrm{i} w$, the Voigt function can be represented in terms of the complex error function $W(z)$ :

$\phi_{V}(z)=\sqrt{\frac{\ln (2)}{\pi}} \frac{1}{\Delta \nu_{\mathrm{D}}} \mathfrak{R}[W(z)]$

which can be computed efficiently by numerous algorithms. In the low pressure limit, where $\Delta v_{\mathrm{D}} \gg \Delta v_{\mathrm{C}}(a \rightarrow 0)$, the Voigt profile renders the Doppler profile, while at higher pressures, when $\Delta v_{\mathrm{C}} \gg \Delta v_{\mathrm{D}}(a \gg 1)$, it reduces to the Lorentz profile.

As can be inferred from Eqs. (A.1) and (A.4), in the Doppler limit the intensity at the line centre will be proportional to pressure (or, equivalently, to number density), and the linewidth will remain constant. On the contrary, in the Lorentz limit, the intensity at the line centre will remain constant, and the linewidth will increase linearly with pressure. From a practical standpoint, there are two main consequences for the experiments described here: on the one hand, increasing the partial pressure of a species beyond a certain value will not increase the signal intensity, although the larger width may allow for a higher degree of smoothing, thereby rendering a higher signal to noise ratio. On the other hand, in a mixture of gases, the peak signal of a minor component may be decreased by the broadening caused by a more abundant species. 